Review

\title{
Crataegus pinnatifida: Chemical Constituents, Pharmacology, and Potential Applications
}

\author{
Jiaqi Wu ${ }^{\dagger}$, Wei Peng ${ }^{\dagger}$, Rongxin Qin and Hong Zhou * \\ Department of Pharmacology, College of Pharmacy, The Third Military Medical University, \\ Chongqing 400038, China \\ $\dagger$ These authors contributed equally to this work. \\ * Author to whom correspondence should be addressed; E-Mail: zhouh64@163.com; \\ Tel./Fax: +86-23-6877-1246.
}

Received: 30 December 2013; in revised form: 22 January 2014 / Accepted: 22 January 2014 / Published: 30 January 2014

\begin{abstract}
Crataegus pinnatifida (Hawthorn) is widely distributed in China and has a long history of use as a traditional medicine. The fruit of $C$. pinnatifida has been used for the treatment of cardiodynia, hernia, dyspepsia, postpartum blood stasis, and hemafecia and thus increasing interest in this plant has emerged in recent years. Between 1966 and 2013, numerous articles have been published on the chemical constituents, pharmacology or pharmacologic effects and toxicology of $C$. pinnatifida. To review the pharmacologic advances and to discuss the potential perspective for future investigation, we have summarized the main literature findings of these publications. So far, over 150 compounds including flavonoids, triterpenoids, steroids, monoterpenoids, sesquiterpenoids, lignans, hydroxycinnamic acids, organic acids and nitrogen-containing compounds have been isolated and identified from $C$. pinnatifida. It has been found that these constituents and extracts of $C$. pinnatifida have broad pharmacological effects with low toxicity on, for example, the cardiovascular, digestive, and endocrine systems, and pathogenic microorganisms, supporting the view that $C$. pinnatifida has favorable therapeutic effects. Thus, although $C$. pinnatifida has already been widely used as pharmacological therapy, due to its various active compounds, further research is warranted to develop new drugs.
\end{abstract}

Keywords: Crataegus pinnatifida; chemical composition; pharmacology; toxicology 


\section{Introduction}

Crataegus pinnatifida, including Crataegus pinnatifida Bge. var. major N. E. Br. and C. pinnatifida Bge, is a traditional, popular Chinese medicinal herb that belongs to the Rosaceae family and is widely distributed in the north of China [1]. Modern investigations have demonstrated that C. pinnatifida has various pharmacological effects on, for example, the cardiovascular, digestive, and endocrine systems, as well as on pathogenic microorganisms [2]. To date, over 150 chemical constituents have been identified from this plant, including flavonoids, triterpenoids, steroids, lignans, organic acids, and nitrogen-containing compounds [3]. Due to its wide spectrum of biological and pharmacological effects, C. pinnatifida has a long history of use as a medicinal plant in China. In the Compendium of Materia Medica (Bencao Gangmu), a famous Traditional Chinese Medicine monograph, the earliest use of dried fruit of $C$. pinnatifida has been described as treatment for cardiodynia, hernia, dyspepsia, postpartum blood stasis, and hemafecia [4]. In addition to being used as a therapeutic medicine, the slightly sour fruit of $C$. pinnatifida is commonly used as a delicious daily food source in China [5].

In the present review we summarize the research advances on the chemical composition, pharmacology and toxicology of $C$. pinnatifida, which will be important for the development of new drugs and full utilization of $C$. pinnatifida. Additionally, we have discussed the potential perspective for future investigations of $C$. pinnatifida.

\section{Chemical Composition}

Research on the chemical components of C. pinnatifida started in the 1960s. Currently, over 150 compositions have been isolated and identified from C. pinnatifida, such as flavonoids, triterpenoids, steroids, monoterpenoids, sesquiterpenoids, lignans, organic acids and nitrogen-containing compounds. In this part, we describe the main chemical constituents of $C$. pinnatifida and their structures (Table 1).

Table 1. Chemical compounds isolated from Chinese Hawthorn.

\begin{tabular}{lllcl}
\hline Classification & No. & Chemical component & Part of Plant & Reference \\
\hline & $\mathbf{1}$ & Apigenin & Leaves & {$[6]$} \\
& $\mathbf{2}$ & Luteolin & Leaves & {$[7]$} \\
& $\mathbf{3}$ & Orientin & Leaves & {$[8]$} \\
& $\mathbf{4}$ & Isoorientin & Leaves & {$[8]$} \\
& $\mathbf{5}$ & Vitexin & Flower & {$[9]$} \\
& $\mathbf{6}$ & Vitexin rhamnoside & Flower & {$[9]$} \\
Flavonoids & $\mathbf{7}$ & Isovitexin & Leaves & {$[10]$} \\
& $\mathbf{8}$ & Hyperoside & Leaves & {$[11]$} \\
& $\mathbf{9}$ & Pinnatifinoside A & Leaves & {$[12]$} \\
& $\mathbf{1 0}$ & Pinnatifinoside B & Leaves & {$[12]$} \\
& $\mathbf{1 1}$ & Pinnatifinoside C & Leaves & {$[12]$} \\
& $\mathbf{1 2}$ & Pinnatifinoside D & Leaves & {$[12]$} \\
& $\mathbf{1 3}$ & Pinnatifinoside I & Leaves & {$[12]$} \\
& $\mathbf{1 4}$ & $3^{\prime \prime \prime}, 4^{\prime \prime \prime}$-di-O-Acetyl-2"-O- $-\alpha$-rhamuosylvitexin & Leaves & {$[13]$} \\
\hline
\end{tabular}


Table 1. Cont.

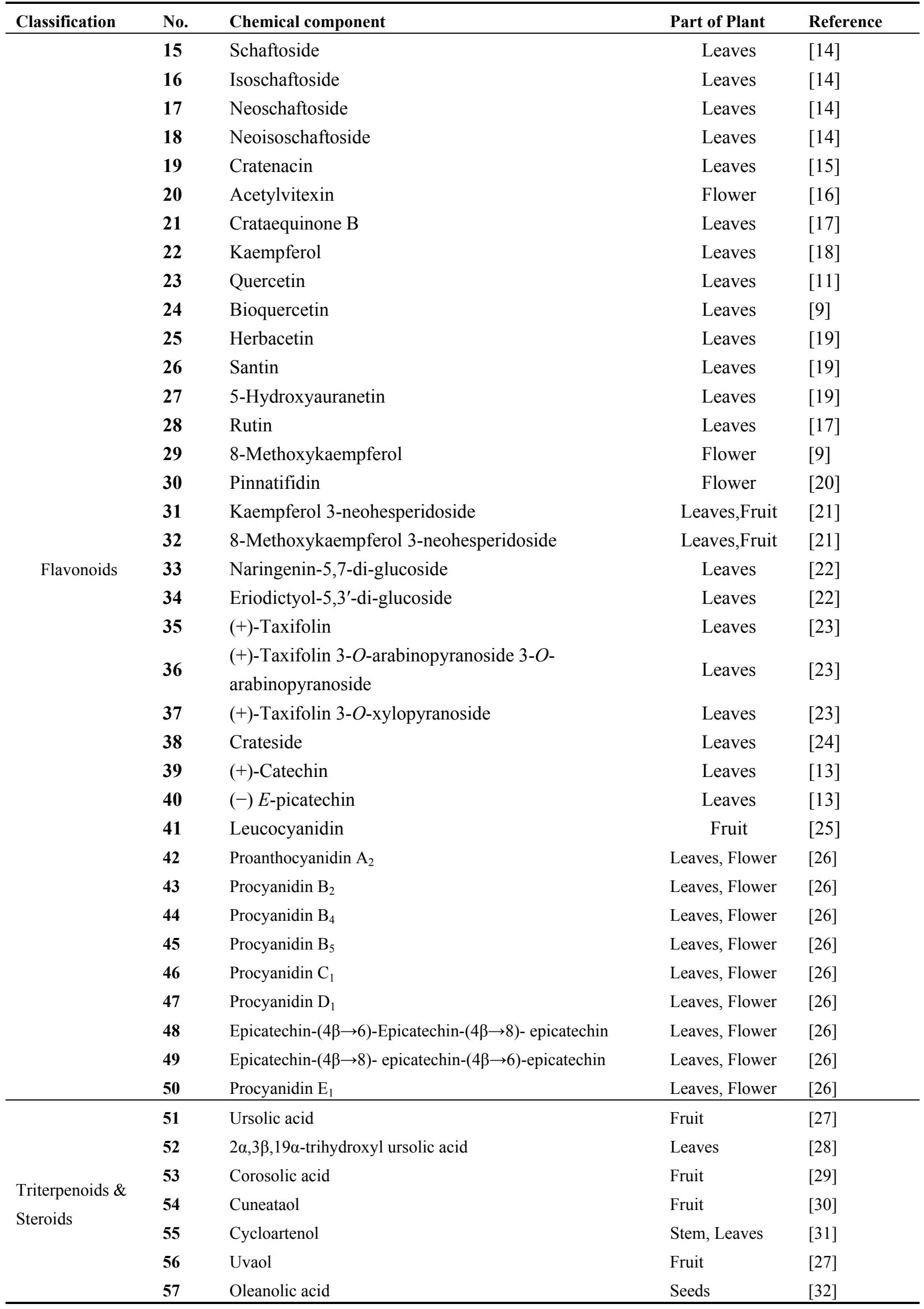


Table 1. Cont.

\begin{tabular}{|c|c|c|c|c|}
\hline Classification & No. & Chemical component & Part of Plant & Reference \\
\hline \multirow{9}{*}{$\begin{array}{l}\text { Triterpenoids \& } \\
\text { Steroids }\end{array}$} & 58 & Maslinic acid & Fruit & [29] \\
\hline & 59 & Butyrospermol & Stem, Leaves & [31] \\
\hline & 60 & 24-Methylene-24-dihydrolanosterol & Stem, Leaves & {$[31]$} \\
\hline & 61 & Betulin & Fruit & {$[27]$} \\
\hline & 62 & $\begin{array}{l}\text { 18,19-seco,2 } \alpha, 3 \beta \text {-Dihydroxy-19-oxo-urs-11,13(18)-dien-28-oic } \\
\text { acid }\end{array}$ & Leaves & {$[33]$} \\
\hline & 63 & $\beta$-Sitosterol & Fruit & [34] \\
\hline & 64 & $\beta$-Daucosterol & Fruit & {$[34]$} \\
\hline & 65 & Stigmosterol & Fruit & {$[34]$} \\
\hline & 66 & 24-Methylene-24-dihydrolanosterol & Stem, Leaves & [31] \\
\hline \multirow{21}{*}{$\begin{array}{l}\text { Monoterpenes \& } \\
\text { sesquiterpenes }\end{array}$} & 67 & 3,9-Dihydroxymegastigma-5-ene & Leaves & {$[33]$} \\
\hline & 68 & $\begin{array}{l}(3 S, 5 R, 6 R, 7 E) \text {-Megatsigmane-7-ene-3-hydroxy-5, 6-epoxy-9-O- } \\
\beta \text {-D-glucopyranoside }\end{array}$ & Leaves & {$[33]$} \\
\hline & 69 & $\begin{array}{l}(3 R, 5 S, 6 S, 7 E, 9 S) \text {-Megastigman-7-ene-3,5,6,9-tetrol 9-O- } \beta \text {-D- } \\
\text { glucopyranoside }\end{array}$ & Leaves & {$[35]$} \\
\hline & 70 & $\begin{array}{l}(6 S, 7 E, 9 R)-6,9-D i h y d r o x y-4,7-m e g a s t i g m a d i e n-3-o n e ~ 9-O-[\beta \text {-D- } \\
\left.\text { xylopyranosyl- }\left(1^{\prime \prime} \rightarrow 6^{\prime}\right)-\beta \text {-D-glucopyranoside }\right]\end{array}$ & Leaves & {$[35]$} \\
\hline & 71 & Linarionoside $\mathrm{C}$ & Leaves & {$[36]$} \\
\hline & 72 & Linarionoside A & Leaves & {$[36]$} \\
\hline & 73 & Linarionoside B & Leaves & {$[36]$} \\
\hline & 74 & $3 \beta$-D-Glucopyranosyloxy- $\beta$-ionone & Leaves & {$[36]$} \\
\hline & 75 & Icariside $\mathrm{B}_{6}$ & Leaves & {$[36]$} \\
\hline & 76 & Pisumionoside & Leaves & {$[36]$} \\
\hline & 77 & $\begin{array}{l}(3 S, 5 R, 6 R, 7 E, 9 R)-3,6-E p o x y-7-m e g a s t i g m e n-5,9-\text { diol-9-O- } \beta \text {-D- } \\
\text { glucopyranoside }\end{array}$ & Leaves & {$[36]$} \\
\hline & 78 & $(6 S, 7 E, 9 R)$-Roseoside & Leaves & [36] \\
\hline & 79 & $(6 R, 9 R)$-3-Oxo- $\alpha$-ionol-9-O- $\beta$-D-glucopyranoside & Leaves & {$[36]$} \\
\hline & 80 & 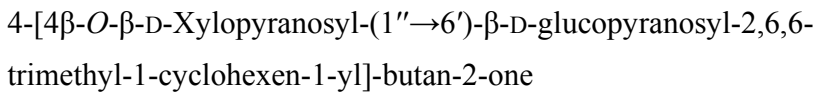 & Leaves & {$[35]$} \\
\hline & 81 & $(3 S, 9 R)$-3,9-Dihydroxy-megastigman-5-ene 3-O-primeveroside & Leaves & {$[35]$} \\
\hline & 82 & $(3 R, 5 S, 6 S, 7 E, 9 S)$-Megastiman-7-ene-3,5,6,9-tetrol & Leaves & {$[35]$} \\
\hline & 83 & $1 \beta, 9 \alpha$-Dihydroxyeudesm-3-en- $5 \beta, 6 \alpha, 7 \alpha, 11 \alpha \mathrm{H}$-12,6-olide & Fruit & {$[37]$} \\
\hline & 84 & $\begin{array}{l}\text { (5Z)-6-[5-(2-Hydroxypropan-2-yl)-2-methyltetrahydrofuran-2-yl] } \\
\text {-3-methylhexa-1,5-dien-3-ol }\end{array}$ & Leaves & {$[35]$} \\
\hline & 85 & $\begin{array}{l}\text { (5Z)-6-[5-(2-O- } \beta \text {-D-Glucopyranosyl-propan-2-yl)-2-methyl } \\
\text { tetrahydrofur-an-2-yl]-3-methylhexa-1,5-dien-3-ol }\end{array}$ & Leaves & {$[35]$} \\
\hline & 86 & $\begin{array}{l}\text { 5-Ethenyl-2-[2-O- } \beta \text {-D-glucopyranosyl- }\left(1^{\prime \prime} \rightarrow 6^{\prime}\right)-\beta \text {-D- } \\
\text { glucopyranosyl-propan-2-yl]-5-methyltetrahydrofuran-2-ol }\end{array}$ & Leaves & {$[35]$} \\
\hline & 87 & Gibberellic acid & Fruit & [38] \\
\hline \multirow{4}{*}{ Lignans } & 88 & $\begin{array}{l}\text { (2,3-Dihydro-2-(4-O- } \beta \text {-D-glueopyranosyl-3-methoxy-Phenyl)-3- } \\
\text { hydroxymethyl-5-(3-hydroxypropyl)-7-methoxybenzofuran) }\end{array}$ & Leaves & [39] \\
\hline & 89 & Shanyenoside A & Leaves & [40] \\
\hline & 90 & $(7 S, 8 R)$-Urolignoside & Leaves & {$[36]$} \\
\hline & 91 & $(-)-2 \mathrm{a}-O-(\beta$-D-Glucopyranosyl)- lyoniresinol & Leaves & {$[36]$} \\
\hline
\end{tabular}


Table 1. Cont.

\begin{tabular}{|c|c|c|c|c|}
\hline \multirow[t]{10}{*}{ Classification } & No. & Chemical component & Part of PLant & Reference \\
\hline & 92 & Tortoside A & Leaves & [36] \\
\hline & 93 & Verbascoside & Leaves & [36] \\
\hline & 94 & Acernikol-4"-O- $\beta$ - D-glucopyranoside & Leaves & {$[36]$} \\
\hline & 95 & $\begin{array}{l}\text { erythro-1-(4-O- } \beta \text {-D-Glucopyranosyl-3-methoxyphenyl)-2-[4- } \\
\text { (3-hydroxypropyl)-2,6-dimethoxyphenoxy]-1,3-propanediol }\end{array}$ & Leaves & {$[36]$} \\
\hline & 96 & $\begin{array}{l}(7 S, 8 R) \text {-5-Methoxydihydrodehydrodiconiferyl alcohol 4-O- } \beta- \\
\text { D-glucopyranoside }\end{array}$ & Leaves & [36] \\
\hline & 97 & Pinnatifidanin C I & Seeds & [41] \\
\hline & 98 & Pinnatifidanin C II & Seeds & [41] \\
\hline & 99 & Pinnatifidanin C III & Seeds & [41] \\
\hline & 100 & Pinnatifidanin C IV & Seeds & [41] \\
\hline \multirow{14}{*}{ Lignans } & 101 & Pinnatifidanin C V & Seeds & [41] \\
\hline & 102 & Pinnatifidanin C VI & Seeds & {$[40]$} \\
\hline & 103 & Pinnatifidanin C VII & Seeds & [41] \\
\hline & 104 & Pinnatifidanin C VIII & Seeds & [41] \\
\hline & 105 & Pinnatifidanin B I & Seeds & [42] \\
\hline & 106 & Pinnatifidanin B II & Seeds & {$[42]$} \\
\hline & 107 & Pinnatifidanin B III & Seeds & [42] \\
\hline & 108 & Pinnatifidanin B IV & Seeds & [42] \\
\hline & 109 & Pinnatifidanin B V & Seeds & [42] \\
\hline & 110 & Pinnatifidanin B VI & Seeds & [42] \\
\hline & 111 & Pinnatifidanin B VII & Seeds & [42] \\
\hline & 112 & Pinnatifidanin B VIII & Seeds & [42] \\
\hline & 113 & Pinnatifidanin B IX & Seeds & [42] \\
\hline & 114 & Chlorogenic acid & Leaves & [19] \\
\hline \multirow{3}{*}{$\begin{array}{l}\text { Hydroxycinnamic } \\
\text { acids }\end{array}$} & 115 & $\beta$-Coumaric acid & Fruit & [38] \\
\hline & 116 & Caffeic acid & Fruit & [38] \\
\hline & 117 & Ferulic acid & Fruit & {$[38$} \\
\hline \multirow{15}{*}{ Organic acids } & 118 & Benzoic acid & Leaves & [33] \\
\hline & 119 & ( $p$-Hydroxyphenyl) benzoic acid & Seed & [43] \\
\hline & 120 & Gallic acid & Seed & [43] \\
\hline & 121 & Protocatechuic acid & Seed & [43] \\
\hline & 122 & Anisic acid & Fruit & [38] \\
\hline & 123 & Vanillic acid & Fruit & [38] \\
\hline & 124 & Syringic acid & Fruit & [38] \\
\hline & 125 & Gentisic acid & Fruit & [38] \\
\hline & 126 & Malic acid & Fruit & [44] \\
\hline & 127 & Citric acid & Fruit & [44] \\
\hline & 128 & Quinic acid & Fruit & [44] \\
\hline & 129 & Pyruvic acid & Fruit & [44] \\
\hline & 130 & Tartaric acid & Fruit & [44] \\
\hline & 131 & Succinic acid & Fruit & [34] \\
\hline & 132 & Fumaric acid & Seed & [32] \\
\hline
\end{tabular}


Table 1. Cont.

\begin{tabular}{|c|c|c|c|c|}
\hline Classification & No. & Chemical component & Part of Plant & Reference \\
\hline \multirow{6}{*}{ Organic acids } & 133 & Ascorbic acid & Shoot & {$[45]$} \\
\hline & 134 & 2-(4-Hydroxy-2-benzyl) malic acid & Seed & {$[32]$} \\
\hline & 135 & Palmitic acid & Fruit & {$[46]$} \\
\hline & 136 & Stearic acid & Fruit & {$[46]$} \\
\hline & 137 & Oleic acid & Fruit & {$[46]$} \\
\hline & 138 & Linoleic acid & Fruit & [46] \\
\hline \multirow{12}{*}{$\begin{array}{l}\text { Nitrogenous } \\
\text { compounds }\end{array}$} & 139 & Isobutylamine & Leaves & {$[47]$} \\
\hline & 140 & Ethylamine & Leaves & [47] \\
\hline & 141 & Dimethylamine & Leaves & {$[47]$} \\
\hline & 142 & Trimethylamine & Leaves & {$[47]$} \\
\hline & 143 & Isoamyl amine & Leaves & {$[47]$} \\
\hline & 144 & Ethanolamine & Leaves & {$[47]$} \\
\hline & 145 & Choline & Leaves & [47] \\
\hline & 146 & Acetylcholine & Leaves & [47] \\
\hline & 147 & Spermindine & Leaves & {$[47]$} \\
\hline & 148 & $O$-Methoxyphenethylamine & Leaves & {$[47]$} \\
\hline & 149 & Tyramine & Leaves & [47] \\
\hline & 150 & Phenylethylamine & Leaves & [47] \\
\hline \multirow{8}{*}{ Other compounds } & 151 & Hentriacontane & Fruit & [27] \\
\hline & 152 & Hexadecanoic acid, octaconsyl ester & Fruit & {$[27]$} \\
\hline & 153 & Eicosanoic acid, octatriacontyl ester & Fruit & {$[27]$} \\
\hline & 154 & Nonacosan-10-ol & Fruit & [27] \\
\hline & 155 & 2,8-Dihydroxy-3,4,7-trimethoxydibenzofuran & Bark, sapwood & [48] \\
\hline & 156 & $\begin{array}{l}\text { (Z)-3-hexenyl- } O \text { - } \beta \text {-D-glucopyranosyl- }\left(1^{\prime \prime} \rightarrow 6^{\prime}\right)-\beta \text {-D- } \\
\text { glucopyranoside }\end{array}$ & Leaves & [35] \\
\hline & 157 & $\begin{array}{l}\text { (Z)-3-Hexenyl- } O-\beta \text {-D-xylopyranosyl- }\left(1^{\prime \prime} \rightarrow 6^{\prime}\right)-\beta \text {-D- } \\
\text { glucopyranoside }\end{array}$ & Leaves & [35] \\
\hline & 158 & $\begin{array}{l}(Z)-3 \text {-Hexenyl- } O-\beta \text {-D-rhamnopyranosyl- }\left(1^{\prime \prime} \rightarrow 6^{\prime}\right)-\beta \text {-D- } \\
\text { glucopyranoside }\end{array}$ & Leaves & [35] \\
\hline
\end{tabular}

\subsection{Flavonoids}

Flavonoids and its derivatives, including flavones, flavonols, flavanones, flavanonols, flavanols and polymers of flavanols, are the most abundant chemical components of $C$. pinnatifida (Figures 1-4).

\subsubsection{Flavones}

Since the first study of $C$. pinnatifida in the 1960s, flavones have been isolated and identified from the leaves and flowers of $C$. pinnatifida. These flavones are a series of compounds whose aglycones are apigenin or luteolin. These flavones include apigenin (1), luteolin (2), orientin (3), iso-orientin (4), vitexin (5), vitexin rhamnoside (6), isovitexin (7), hyperoside (8), pinnatifinoside A-D, I (9-13), 3"', 4 "'-di-O-acetyl-2"'-O- $\alpha$-rhamnosylvitexin (14), schaftoside (15), isoschaftoside (16), neoschaftoside 
(17), neoisoschaftoside (18), cratenacin (19), acetylvitexin (20). Additionally, crataequinone B (21) was isolated from the leaves of C. pinnatifida [17] (Figure 1).

Figure 1. Chemical structures of flavones in C. pinnatifida.
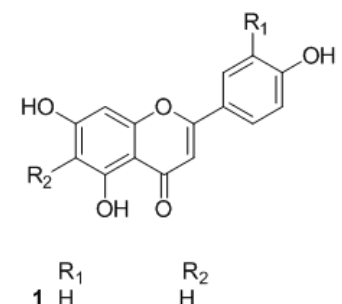

$1 \mathrm{H}$
$2 \mathrm{OH}$

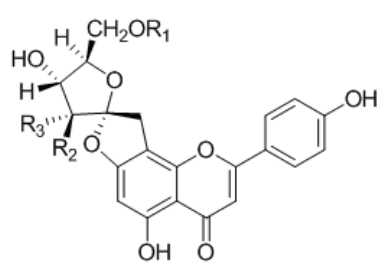

$\begin{array}{llll} & \mathrm{R}_{1} & \mathrm{R}_{2} & \mathrm{R}_{3} \\ \mathbf{9} & \mathrm{H} & \mathrm{OH} & \mathrm{H} \\ 10 & \text { Acetyl } & \mathrm{OH} & \mathrm{H} \\ 11 & \text { Acetyl } & \mathrm{H} & \mathrm{OH}\end{array}$

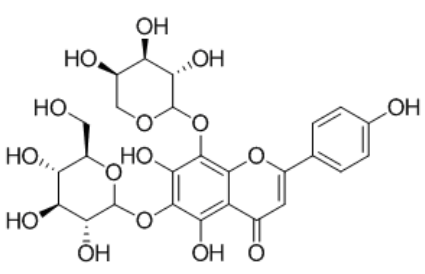

15

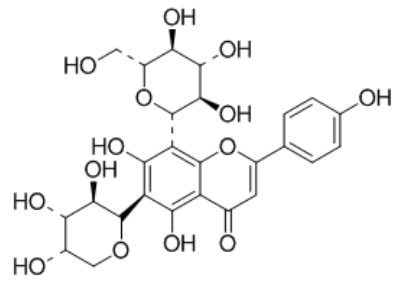

18

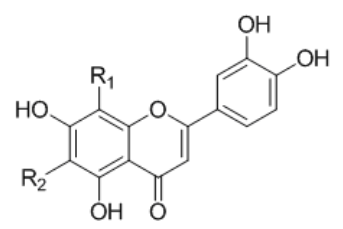

$\begin{array}{ccc} & \mathrm{R}_{1} & \mathrm{R}_{2} \\ 3 & -\mathrm{Glc} & \mathrm{H} \\ 4 & \mathrm{H} & -\mathrm{Glc}\end{array}$

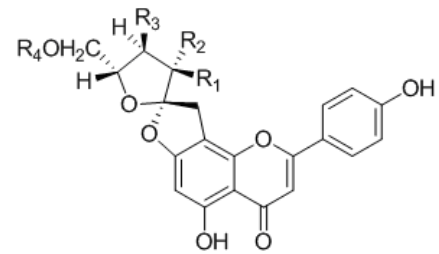

$\begin{array}{lllll} & \mathrm{R}_{1} & \mathrm{R}_{2} & \mathrm{R}_{3} & \mathrm{R}_{4} \\ 12 & \mathrm{OH} & \mathrm{H} & \mathrm{OH} & \text { Acetyl } \\ 13 & \mathrm{H} & \mathrm{OH} & \text { Acetyl } & \mathrm{H}\end{array}$

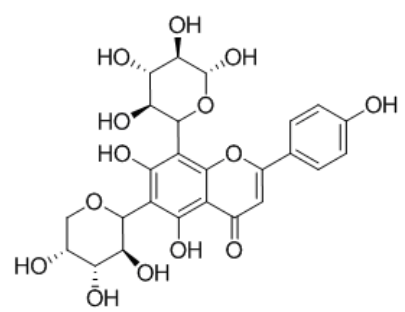

16

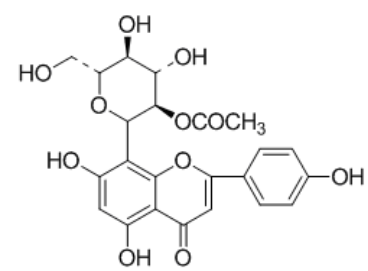<smiles>O=C1C(=O)C2=C3C(O)=CC=CC1=C3OC(O)=C2O</smiles>

21

\subsubsection{Flavonols}

Flavonols are also as abundant in C. pinnatifida as flavones. The configuration of flavonols of C. pinnatifida is mainly quercetin-and kaempferol-like, including kaempferol (22), quercetin (23), bioquercetin (24), herbacetin (25), santin (26), 5-hydroxyauranetin (27), rutin (28), 8-methoxykaempferol (29), pinnatifidin (30), kaempferol 3-neohesperidoside (31), and 8-methoxykaempferol 3-neohesperidoside (32) (Figure 2). 
Figure 2. Chemical structures of flavonols in C. pinnatifida.<smiles>[R]c1ccc(-c2oc3c([R])c([R])c([R])c(O)c3c(=O)c2[R5])cc1[R]</smiles><smiles>[R]Oc1c(-c2ccc(O)cc2)oc2c(OC)c(O)cc(O)c2c1=O</smiles>

$\begin{array}{llllllll} & \mathrm{R}_{1} & \mathrm{R}_{2} & \mathrm{R}_{3} & \mathrm{R}_{4} & \mathrm{R}_{5} & \mathrm{R}_{6} & \text { 29 R=H } \\ \mathbf{2 2} & \mathrm{H} & \mathrm{OH} & \mathrm{OH} & \mathrm{H} & \mathrm{OH} & \mathrm{H} & \mathbf{3 0} \mathrm{R}=-\mathrm{Glc} \\ \mathbf{2 3} & \mathrm{OH} & \mathrm{OH} & \mathrm{OH} & \mathrm{H} & \mathrm{OH} & \mathrm{H} & \\ \mathbf{2 4} & \mathrm{OH} & \mathrm{OH} & -\mathrm{O}-\text {-robinobioside } & \mathrm{H} & \mathrm{OH} & \mathrm{H} & \\ \mathbf{2 5} & \mathrm{H} & \mathrm{OH} & \mathrm{OH} & \mathrm{H} & \mathrm{OH} & \mathrm{OH} & \\ \mathbf{2 6} & \mathrm{H} & \mathrm{OCH}_{3} & \mathrm{OCH}_{3} & \mathrm{OCH}_{3} & \mathrm{OH} & \mathrm{H} & \\ \mathbf{2 7} & \mathrm{H} & \mathrm{OCH}_{3} & \mathrm{OCH}_{3} & \mathrm{OCH}_{3} & \mathrm{OCH}_{3} & \mathrm{OCH}_{3} & \\ \mathbf{2 8} & \mathrm{OH} & \mathrm{OH} & \text {-O-rutinose } & \mathrm{H} & \mathrm{OH} & \mathrm{H} & \end{array}$<smiles>[R2]c1c(O)cc(O)c2c(=O)c(O[C@H]3O[C@H](O)[C@@H](O)[C@H](O)[C@H]3O[C@H]3O[C@H](C)[C@@H](O)[C@H](O)[C@H]3O)c(-c3ccc(O)cc3)oc12</smiles>

$31 \mathrm{R}=\mathrm{H}$ $32 \mathrm{R}=\mathrm{OCH}_{3}$

\subsubsection{Flavanones and Flavanonols}

In 1971, naringenin 5,7-diglucoside (33) and eriodictyol-5,3'-diglucoside (34) were isolated from the leaves of $C$. pinnatifida [22]. Later, [(+)-taxifolin (35)], [(+)-taxifolin-3-O-arabinopyranoside (36)] and $[(+)$-taxifolin 3-O-xylopyranoside (37)] were also isolated from the leaves [23]. Additionally, crateside (38) was isolated [24] (Figure 3).

Figure 3. Chemical structures of flavanones and flavanonols.<smiles>[R20]Oc1cc([R20])c2c(c1)O[C@H](c1ccc(O)c([R])c1)CC2=O</smiles><smiles>[R20]Oc1cc([C@H]2Oc3cc(O)cc(O)c3C(=O)[C@@H]2O[R2])ccc1O</smiles>

$$
\begin{array}{cclc} 
& \mathrm{R}_{1} & \mathrm{R}_{2} & \mathrm{R}_{3} \\
33 & -\mathrm{Glc} & -\mathrm{Glc} & \mathrm{H} \\
\mathbf{3 4} & \text {-Glc } & \mathrm{H} & -\mathrm{Glc}
\end{array}
$$

$\begin{array}{lll} & \mathrm{R}_{1} & \mathrm{R}_{2} \\ 35 & \mathrm{H} & \mathrm{H} \\ 36 & \text { Ara } & \mathrm{H} \\ \mathbf{3 7} & -\mathrm{Xyl} & \mathrm{H} \\ \mathbf{3 8} & \mathrm{H} & \text {-Ara }\end{array}$

\subsubsection{Flavanols and the Polymers of Flavanols}

Flavanols and flavanol polymers, the elementary units of which were (+)-catechin (39)], (-)-Epicatechin (40) and leucocyanidin (41), are also abundant in C. pinnatifida $[13,25]$. The polymers are a series of compounds where these three compounds are polymerized [26]. Dimers include proanthocyanidin A2 (42), procyanidin B2, B4, B5 (43-45), and trimers include procyanidin $\mathrm{C} 1$ (46),

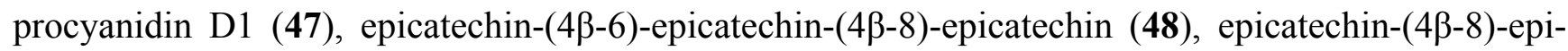

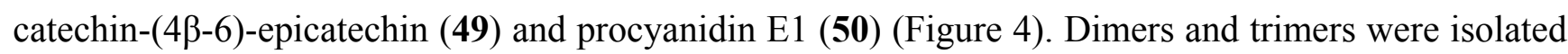
and identified from C. pinnatifida in 2002. 
Figure 4. Chemical structures of flavanols and the polymers of flavanols in C. pinnatifida.

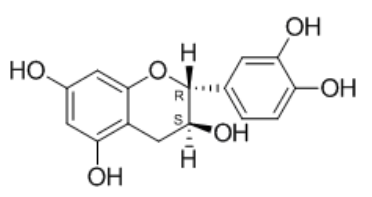

39

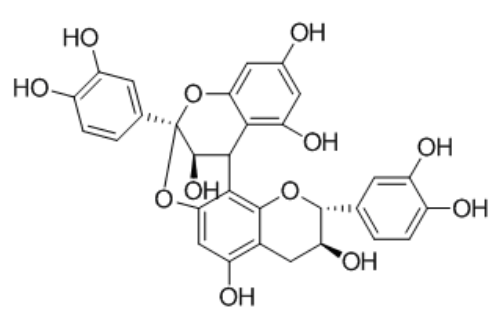

42

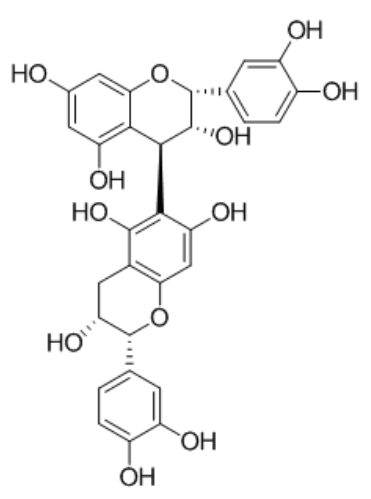

45

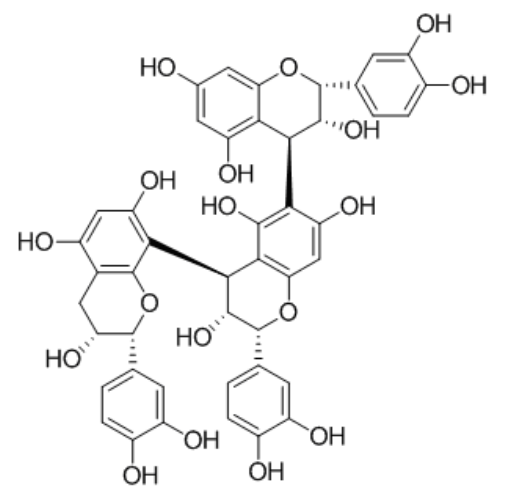

48

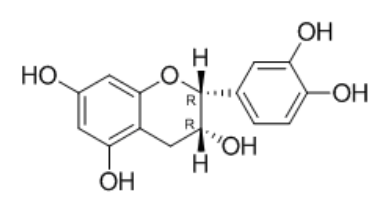

40

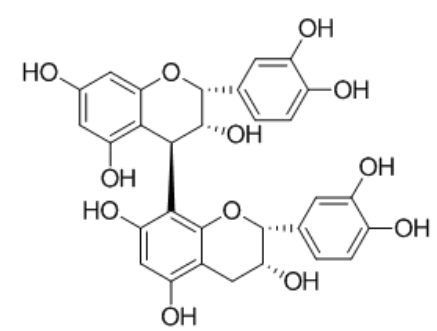

43

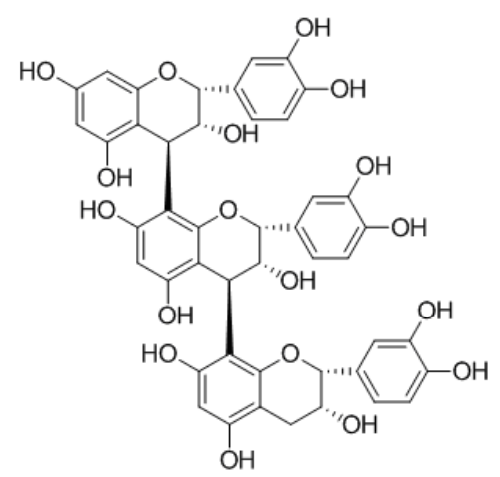

46

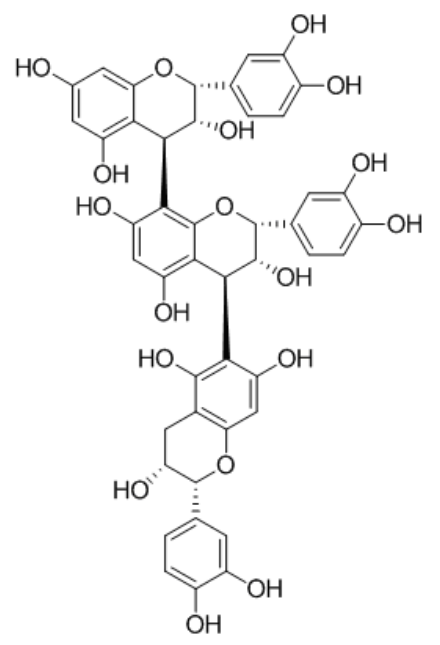

49

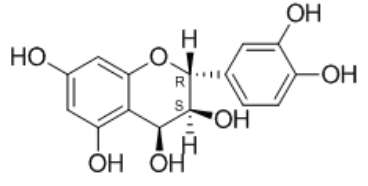

41

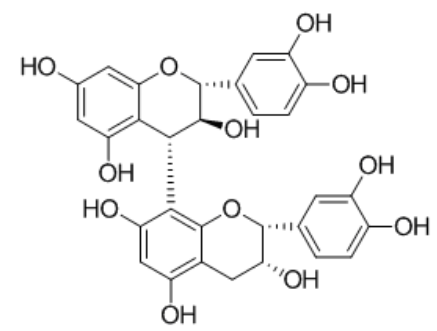

44<smiles>Oc1cc(O)c2c(c1)O[C@H](c1ccc(O)c(O)c1)[C@H](O)[C@H]2c1c(O)cc(O)c2c1O[C@H](c1ccc(O)c(O)c1)[C@H](O)[C@H]2c1c(O)cc(O)c2c1O[C@H](c1ccc(O)c(O)c1)[C@H](O)[C@H]2c1c(O)cc(O)c2c1O[C@H](c1ccc(O)c(O)c1)[C@H](O)C2</smiles>

47

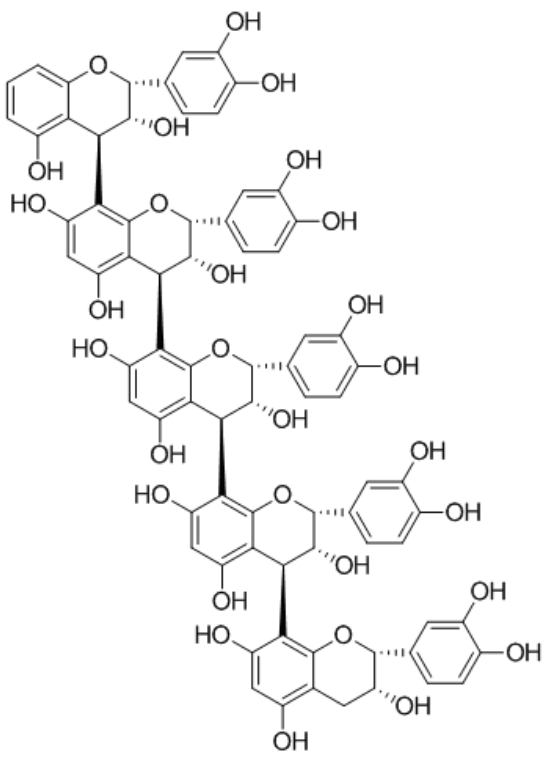

50 


\subsection{Triterpenoids and Steroids}

\subsubsection{Triterpenoids}

Since the first study of $C$. pinnatifida in the 1960s, triterpenoids and their derivatives have been isolated and identified. These triterpenoids are classified into tetracyclic triterpenoids and pentacyclic triterpenoids, such as ursolic acid (51), 2 $\alpha, 3 \beta, 19 \alpha$-trihydroxyursolic acid (52), corosolic acid (53), cuneataol (54), cycloartenol (55), uvaol (56), oleanolic acid (57), crataegolic acid (58), butyrospermol (59), 24-methylene-24-dihydrolanosterol (60), betulin (61), and 18,19-seco-2 $\alpha, 3 \beta$-dihydroxy-19-oxo-urs11,13(18)-dien-28-oic acid (62) [27-33] (Figure 5).

Figure 5. Chemical structures of triterpenoids in C. pinnatifida.

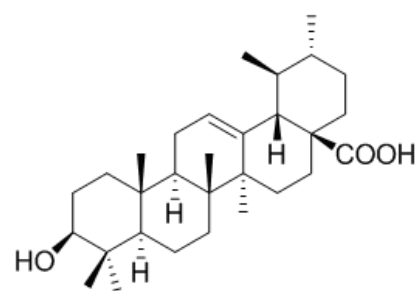

51

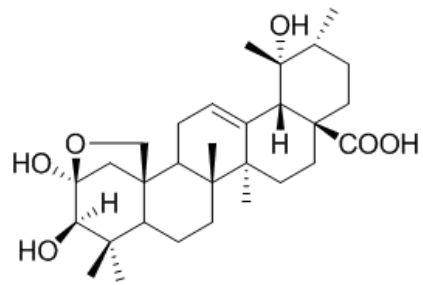

54

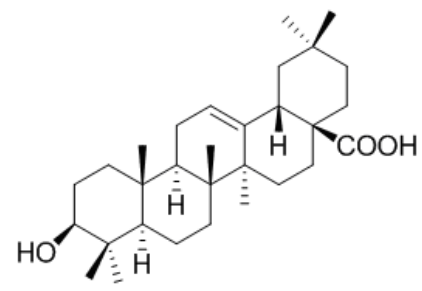

57

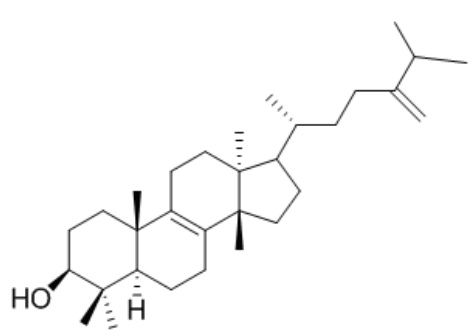

60

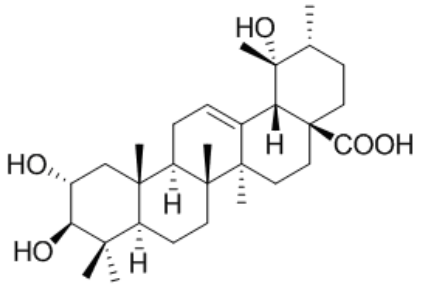

52

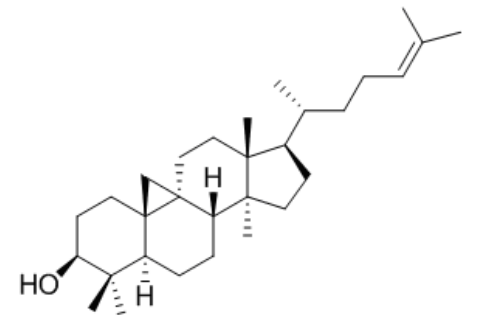

55

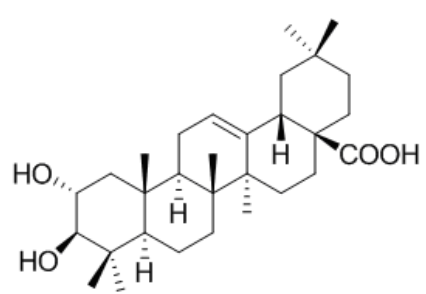

58

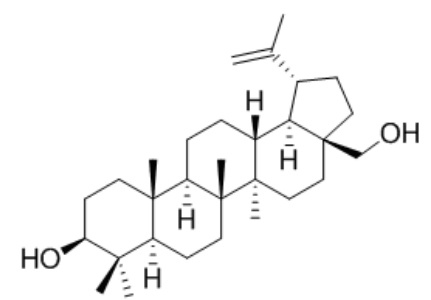

61

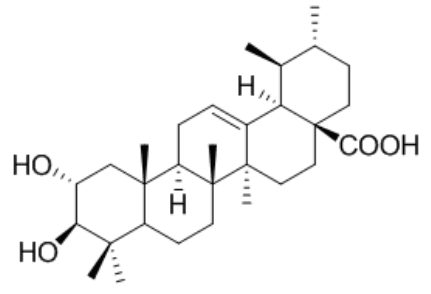

53

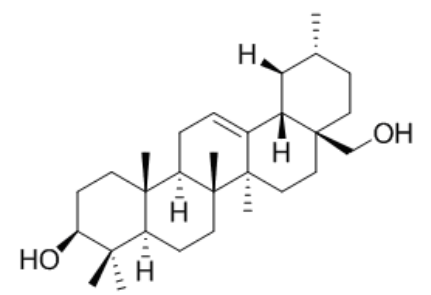

56

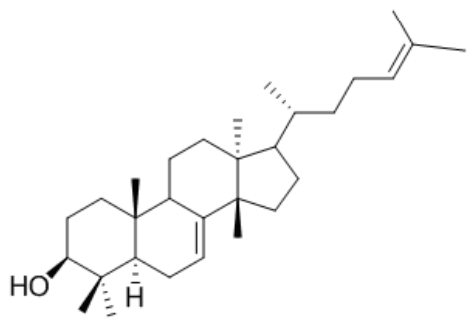

59

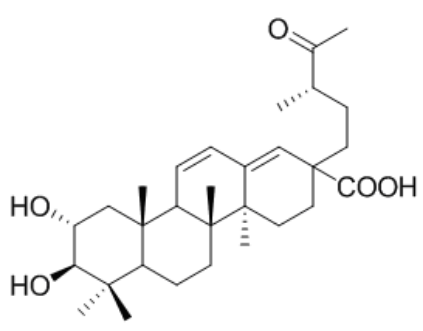

62 


\subsubsection{Steroids}

So far, four steroids were isolated from C. pinnatifida. In 1997, 24-methylen-24-dihydrolanosterol (66) was isolated from stems and leaves of $C$. pinnatifida [31]. Later, $\beta$-sitosterol (63), $\beta$-daucosterol (64), stigmosterol (65) were isolated from fruits of C. pinnatifida [34] (Figure 6).

Figure 6. Chemical structures of steroids in C. pinnatifida.

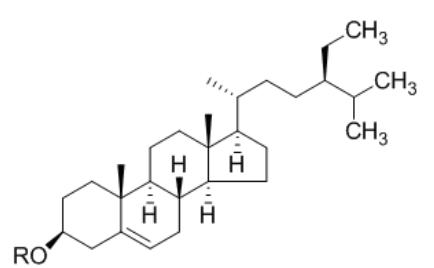

$63 \mathrm{R}=\mathrm{H}$ $64 \mathrm{R}=-\mathrm{Glc}$

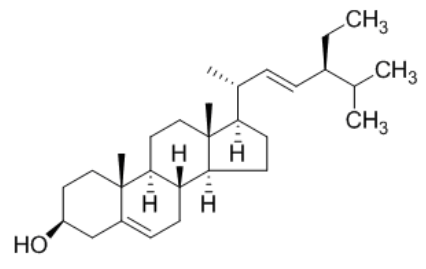

65

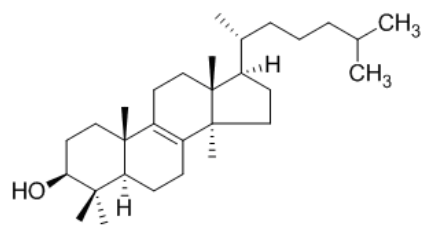

66

\subsection{Monoterpenoids and Sesquiterpenoids}

Monoterpenoids and sesquiterpenoids are the main constituents of the volatile oil from C. pinnatifida, which is an important raw material in the spice and medical industry. Monoterpenoids and sesquiterpenoids are also abundant in the leaves of $C$. pinnatifida. 3,9-Dihydroxymegastigma-5ene (67) and $(3 S, 5 R, 6 R, 7 E)$-megatsigmane-7-ene-3-hydroxy-5,6-epoxy-9-O- $\beta$-D-glucopyranoside (68) were new compounds firstly isolated from the leaves of $C$. pinnatifida and identified in 2010 [33]. Later, $(3 R, 5 S, 6 S, 7 E, 9 S)$-megastigman-7-ene-3,5,6,9-tetrol-9-O- $\beta$-D-glucopyranoside (69) and $(6 S, 7 E$, 9R)-6,9-dihydroxy-4,7-megastigmadien-3-one 9- $O$-[ $\beta$-D-xylopyranosyl-(1" $\left.\rightarrow 66^{\prime}\right)-\beta$-D-glucopyranoside] (70) were isolated and identified from C. pinnatifida leaves in 2011 [35]. Additionally, linarionoside $\mathrm{C}$ (71), linarionoside A, B (72-73), 3 $\beta$-glucopyranosyloxy- $\beta$-ionone (74), icariside B6 (75), pisumionoside (76), (3S,5R,6R,7E,9R)-3,6-epoxy-7-megastigmen-5,9-diol-9-O- $\beta$-D-glucopyranoside (77), $(6 S, 7 E, 9 R)$-roseoside (78) and $(6 R, 9 R)$-3-oxo- $\alpha$-ionol-9- $O$ - $\beta$-D-glucopyranoside (79) have been isolated from the leaves of $C$. pinnatifida in 2010 [36] (Figure 7).

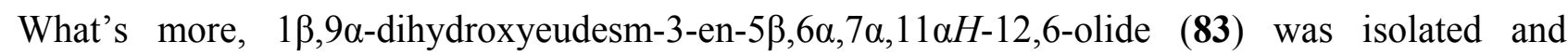
identified from the fruits of $C$. pinnatifida [37]. In addition, six others were subsequently isolated from

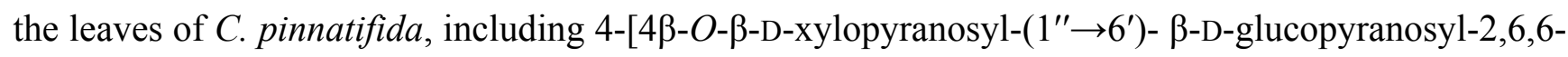
trimethyl-1-cyclohexen-1-yl]-butan-2-one $\quad(\mathbf{8 0}), \quad(3 S, 9 R)-3,9-d i h y d r o x y m e g a s t i g m a n-5-e n e \quad 3-O-$ primeveroside (81), (3R,5S,6S,7E,9S)-megastiman-7-ene-3,5,6,9-tetrol (82), (5Z)-6-[5-(2-hydroxypropan2-yl)-2-methyltetrahydrofuran-2-yl]-3-methylhexa-1,5-dien-3-ol (84), (5Z)-6-[5-(2-O- $\beta$-D-glucopyranosylpropan-2-yl)-2-methyltetrahydrofuran-2-yl]-3-methylhexa-1,5-dien-3-ol (85), 5-ethenyl-2-[2-O- $\beta$-Dglucopyranosyl-(1" $\left.\rightarrow 6^{\prime}\right)-\beta$-D-glucopyranosyl-propan-2-yl]-5-methyltetrahydrofuran-2-ol (86) [35], and gibberellic acid (87) [38] (Figure 7). 
Figure 7. Chemical structures of monoterpenoids and sesquiterpenoids in C. pinnatifida.<smiles>CC1=C(CC[C@@H](C)O)C(C)(C)CC(O)C1</smiles>

67<smiles>[R20]OC(C)CCC1=C(C)CC([R20])CC1(C)C</smiles>

$\begin{array}{rr}R_{1} & R_{2} \\ 72-G l C & H \\ 73 & -G l c\end{array}$

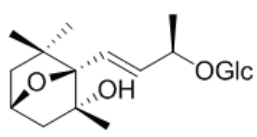

77<smiles>CC(C=CC1(C)[C@@](C)(O)CC(O)C[C@]1(C)O)OCl</smiles>

68<smiles>CC(=O)/C=C/C1=C(C)C[C@@H](OCl)CC1(C)C</smiles>

74<smiles>CC1=CC(=O)CC(C)(C)C1(O)/C=C/C(O)O[Cl+3]</smiles>

78<smiles>[R]OC(C)/C=C/[C@@]1(O)[C@@H](O)C[C@@H](O)CC1(C)C</smiles>

$69 \mathrm{R}=\mathrm{H}$

$70 \mathrm{R}=-\mathrm{Glc}$<smiles>CC(=O)CCC1=C(C)C[C@@H](OC2CCCCC2)CC1(C)C</smiles>

75<smiles>COC(C)/C=C/C1C(C)=CC(=O)CC1(C)C</smiles>

79<smiles>CC1=C(CCC(C)OC(C)(C)C)C(C)(C)CC(O[GeH3])C1</smiles>

71

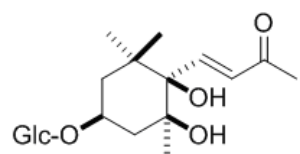

76

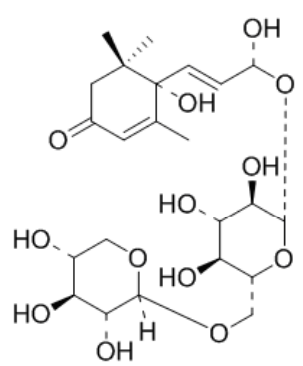

82<smiles>C=C[C@]1(C)CC[C@]2(O[C@@H]3C(OC(COC4CCCCC4)C(O)C3O)OC2(C)C)O1</smiles>

86

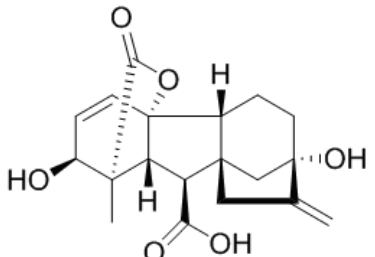

87<smiles>CC1=CCC(O)[C@]2(O)C[C@H](O)C3CC(C)C(=O)OC132</smiles>

83

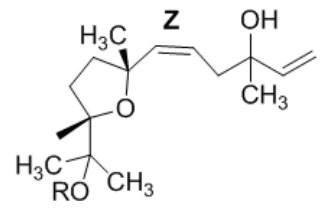

$84 \mathrm{R}=\mathrm{H}$ $85 \mathrm{R}=-\mathrm{Glc}$

\subsection{Lignans}

Lignans, a kind of natural product containing two phenylpropane frameworks, is another characteristic component of $C$. pinnatifida, where they mainly exist in the leaves. A new identified compound, shanyenoside (A) (89), was isolated from the leaves of C. pinnatifida in 2006 [40]. In 2009, (2,3-dihydro-2-(4-O- $\beta$-D-glueopyranosyl-3-methoxyphenyl)-3-hydroxymethyl-5-(3-hydroxypropyl)-7methoxybenzofuran) (88) was isolated from the leaves of $C$. pinnatifida [39]. Later, (7S,8R)urolignoside (90), (-)-2a-O-( $\beta$-D-glucopyranosyl)lyoniresinol (91), tortoside A (92), verbascoside (93), acernikol-4"-O- $\beta$-D-glucopyranoside (94), erythro-1-(4-O- $\beta$-D-glucopyranosyl-3-methoxyphenyl)-2- 
[4-(3-hydroxypropyl)-2,6-dimethoxyphenoxy]-1,3-propanediol (95) and (7S,8R)-5-methoxydihydrodehydrodiconiferyl alcohol 4- $O$ - $\beta$-D-glucopyranoside (96) were also isolated from the leaves of C. pinnatifida in 2010 [36]. Recently, some novel neolignans were isolated from the seeds of C. pinnatifida, including pinnatifidanin C I-VIII, pinnatifidanin B I-IX (97-112) [40-42] (Figure 8).

\subsection{Hydroxycinnamic Acids}

Lots of hydroxycinnamic acids were also isolated from the leaves and fruits of $C$. pinnatifida, including chlorogenic acid (114), $\beta$-coumaric acid (115), caffeic acid (116), and ferulic acid (117) [19,38] (Figure 9).

Figure 8. Chemical structures of lignans in C. pinnatifida.

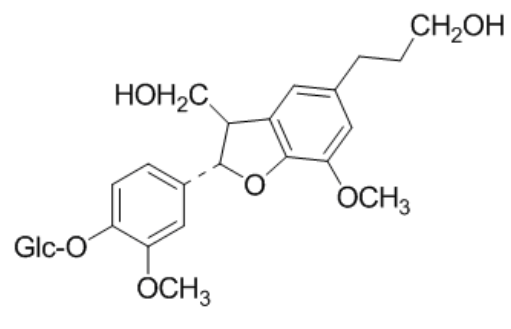

88

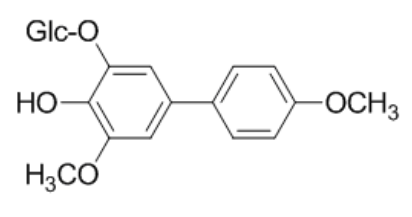

89<smiles>COc1cc([C@H]2Oc3c(O)cc(CCCO)cc3[C@@H]2CO)ccc1OC1CCCCC1</smiles>

90<smiles>COc1cc([C@H]2c3c(cc(OC)c(O)c3OC)C[C@@H](COCl)[C@@H]2CO)cc(OC)c1O</smiles>

91<smiles>COc1cc(C2OCC3C2CO[C@@H]3c2cc(OC)c(OC)c(OC)c2)cc(OC)c1O</smiles>

92

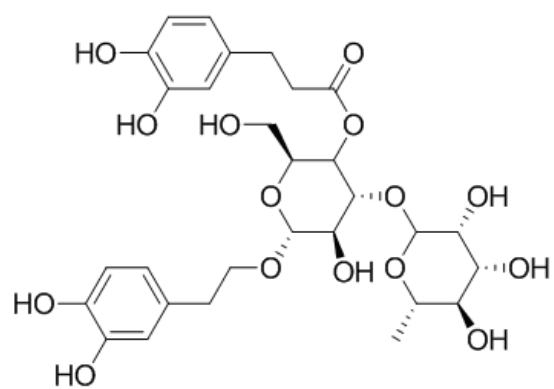

93<smiles>COc1cc([C@@H](O)C(CO)Oc2c(OC)cc([C@@H]3Oc4c(OC)cc(CCCO)cc4[C@@H]3CO)cc2OC)ccc1OC(C)=O</smiles><smiles>COc1cc(CCCO)cc(OC)c1OC(CO)[C@H](C)O</smiles><smiles>COc1cc([C@H]2Oc3c(OC)cc(CCCO)cc3[C@@H]2CO)cc(OC)c1OC1CCCCC1</smiles> 
Figure 8. Cont.<smiles>[R]C(CO)C(=O)c1cc(OC)c2c(c1)C(CO)C(c1cc(O)cc(OC)c1)O2</smiles><smiles>COc1cc(O)cc(C2Oc3c(OC)cc(O)cc3C2CO)c1</smiles><smiles>[R]C(CO)[C@H]([R])c1cc(OC)c(O[C@H](CO)[C@@H]([R])c2cc(O)cc(OC)c2)c(OC)c1</smiles>

$106 \mathrm{R}_{1}=\mathrm{OCH}_{3} ; \mathrm{R}_{2}=\mathrm{H} ; \mathrm{R}_{3}=\mathrm{OH}$; erthro $-7 S, 8 R$

$107 \mathrm{R}_{1}=\mathrm{OCH}_{3} ; \mathrm{R}_{2}=\mathrm{OH} ; \mathrm{R}_{3}=\mathrm{OH}$; erthro-7S, 8R; 7', 8'-erythro

$108 \mathrm{R}_{1}=\mathrm{OCH}_{2} \mathrm{CH}_{3} ; \mathrm{R}_{2}=\mathrm{OH} ; \mathrm{R}_{3}=\mathrm{H}$; erthro- $7 \mathrm{~S}, 8 R$

$109 \mathrm{R}_{1}=\mathrm{OCH}_{2} \mathrm{CH}_{3} ; \mathrm{R}_{2}=\mathrm{OH} ; \mathrm{R}_{3}=\mathrm{H}$; erthro- $7 R, 8 \mathrm{~S}$<smiles>[R]C(CO)C([R])c1cc(OC)c2c(c1)C(CO)C(c1cc(O)cc(OC)c1)O2</smiles>

$99 \mathrm{R}_{1}=\mathrm{OCH}_{3} ; \mathrm{R}_{2}=\mathrm{OH} ; 7 S, 8 R ; 7^{\prime}, 8^{\prime}$-threo

$100 \mathrm{R}_{1}=\mathrm{OCH}_{3} ; \mathrm{R}_{2}=\mathrm{OH} ; 7 R, 8 S ; 7^{\prime}, 8^{\prime}$-threo

$101 \mathrm{R}_{1}=\mathrm{OCH}_{2} \mathrm{CH}_{3} ; \mathrm{R}_{2}=\mathrm{OH} ; 7 S, 8 R ; 7^{\prime}, 8^{\prime}$-threo

$103 \mathrm{R}_{1}=\mathrm{OCH}_{2} \mathrm{CH}_{3} ; \mathrm{R}_{2}=\mathrm{OH} ; 7 R, 8 S ; 7^{\prime}, 8^{\prime}$-threo

$104 \mathrm{R}_{1}=\mathrm{OCH}_{2} \mathrm{CH}_{3} ; \mathrm{R}_{2}=\mathrm{H} ; 7 R, 8 \mathrm{~S}$<smiles>[R]c1cc(CCCO)ccc1O[C@H](CO)[C@@H]([R])c1ccc(O)c(OC)c1</smiles>

$110 \mathrm{R}_{1}=\mathrm{OCH}_{3} ; \mathrm{R}_{2}=\mathrm{OCH}_{3} ;$ terthro- $7 R, 8 R$ $111 \mathrm{R}_{1}=\mathrm{OCH}_{3} ; \mathrm{R}_{2}=\mathrm{OCH}_{3} ;$ erthro- $7 \mathrm{~S}, 8 R$<smiles>[R]C(c1ccc(O)c(OC)c1)[C@@H](CO)Oc1ccc(/C=C/C=O)cc1OC</smiles>

$112 \mathrm{R}=\mathrm{OCH}_{3} ;$ terthro- $7 R, 8 R ; 7^{\prime} E$<smiles>[R]C/C=C/c1ccc(O[C@H](CO)[C@@H](OC)c2ccc(O)c(OC)c2)c(OC)c1</smiles>

Figure 9. Chemical structures of hydroxycinnamic acids in C. pinnatifida.<smiles>O=C(/C=C/c1ccc(O)c(O)c1)OC1C[C@H](O)C[C@](O)(C(=O)O)C1</smiles>

114

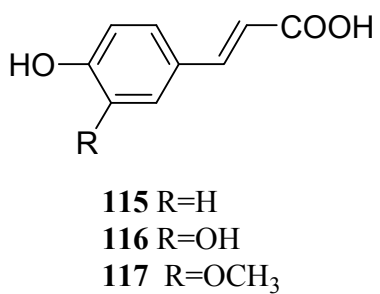

\subsection{Organic Acids}

Organic acids of $C$. pinnatifida mainly include phenolic acids and other organic acids. Phenolic acids include benzoic acid (118), ( $p$-hydroxyphenyl)benzoic acid (119), gallic acid (120), protocatechuic acid (121), anisic acid (122), vanillic acid (123), syringic acid (124), gentisic acid (125) [19,33,40,41]. Other organic acids include malic acid (126), citric acid (127), quinic acid (128), pyruvic acid (129), tartaric acid (130), succinic acid (131), fumaric acid (132), ascorbic acid (133), 
2-(4-hydroxy2 benzyl) malic acid (134), palmitic acid (135), stearic acid (136), oleic acid (137), and linoleic acid (138) [32,34,38,43-46] (Figure 10).

Figure 10. Chemical structures of organic acids in C. pinnatifida.<smiles>[R]Cc1c([R])c([R])c(C(=O)O)c([R])c1[R]</smiles><smiles>O=C(O)CC(O)C(=O)O</smiles>

126

$\begin{array}{llllll} & \mathrm{R}_{1} & \mathrm{R}_{2} & \mathrm{R}_{3} & \mathrm{R}_{4} & \mathrm{R}_{5} \\ 118 & \mathrm{H} & \mathrm{H} & \mathrm{H} & \mathrm{H} & \mathrm{H} \\ 119 & \mathrm{H} & \mathrm{H} & \mathrm{OH} & \mathrm{H} & \mathrm{H} \\ 120 & \mathrm{H} & \mathrm{OH} & \mathrm{OH} & \mathrm{OH} & \mathrm{H} \\ 121 & \mathrm{H} & \mathrm{H} & \mathrm{OH} & \mathrm{OH} & \mathrm{H} \\ 122 & \mathrm{H} & \mathrm{H} & \mathrm{OCH}_{3} & \mathrm{H} & \mathrm{H} \\ 123 & \mathrm{H} & \mathrm{OCH}_{3} & \mathrm{OH} & \mathrm{H} & \mathrm{H} \\ 124 & \mathrm{H} & \mathrm{OCH}_{3} & \mathrm{OH} & \mathrm{OCH} & \mathrm{H} \\ 125 & \mathrm{OH} & \mathrm{H} & \mathrm{H} & \mathrm{H} & \mathrm{OH}\end{array}$<smiles>O=C(O)C(O)C(O)C(=O)O</smiles>

130<smiles>O=C(O)CCC(=O)O</smiles>

131<smiles>O=C(O)C1(O)C[C@H](O)[C@H](O)C[C@H]1O</smiles>

128<smiles>O=C(O)/C=C/C(=O)O</smiles>

132<smiles>O=C(O)CC(O)(CC(=O)O)C(=O)O</smiles>

127<smiles>CC(=O)C(=O)O</smiles>

129<smiles>O=C1OC([C@@H](O)CO)C(O)=C1O</smiles>

133<smiles>O=C(O)CC(Oc1ccc(O)cc1)C(=O)O</smiles><smiles>CCCCCCCCCCCCCCC(=O)O</smiles>

135<smiles>CCCCCCCCCCCCCCCCCC(=O)O</smiles>

136

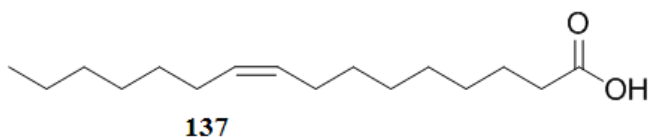

137

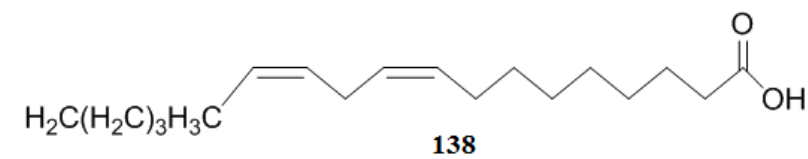

\subsection{Nitrogen-containing Compounds}

So far, twelve nitrogen-containing compounds were isolated from the leaves of $C$. pinnatifida. In 1990, the nitrogen-containing compounds isobutylamine (139), ethylamine (140), dimethylamine (141), trimethylamine (142), isoamylamine (143), ethanolamine (144), choline (145), acetylcholine (146), spermindine (147), O-methoxyphenethylamine (148), tyramine (149), and phenylethylamine (150) were isolated and identified [47] (Figure 11).

\subsection{Others}

A compound identified as 2,8-dihydroxy-3,4,7-trimethoxydibenzofuran (151) was isolated from the bark and sapwood of C. pinnatifida [48]. Hentriacontane (152), (hexadecanoic acid, octaconsyl ester) (153), (eicosanoic acid, octatriacontyl ester) (154) and nonacosan-10-ol (155) were also isolated from 
the fruits of $C$. pinnatifida [27]. Recently, three new compounds, ( $Z$ )-3-hexenyl- $O$ - $\beta$-D-glucopyranosyl- $\left(1^{\prime \prime} \rightarrow 6^{\prime}\right)-\beta$-D-glucopyranoside $\quad(\mathbf{1 5 6}), \quad(Z)-3$-hexenyl- $O-\beta$-D-xylopyranosyl- $\left(1^{\prime \prime} \rightarrow 6^{\prime}\right)-\beta$-Dglucopyranoside (157), and (Z)-3-hexenyl-O- $\beta$-D-rhamnopyranosyl-( $\left(1^{\prime \prime} \rightarrow 6^{\prime}\right)$ - $\beta$-D-glucopyranoside (158) were isolated from the leaves of $C$. pinnatifida [35] (Figure 12). Additionally, plenty of sugars and sugar alcohols were found in the C. pinnatifida, including glucose, sucrose, fructose, sorbitol, and myoinositol, with fructose being the most abundant sugar in the fruits [49].

Figure 11. Chemical structures of nitrogen-containing compounds in C. pinnatifida.

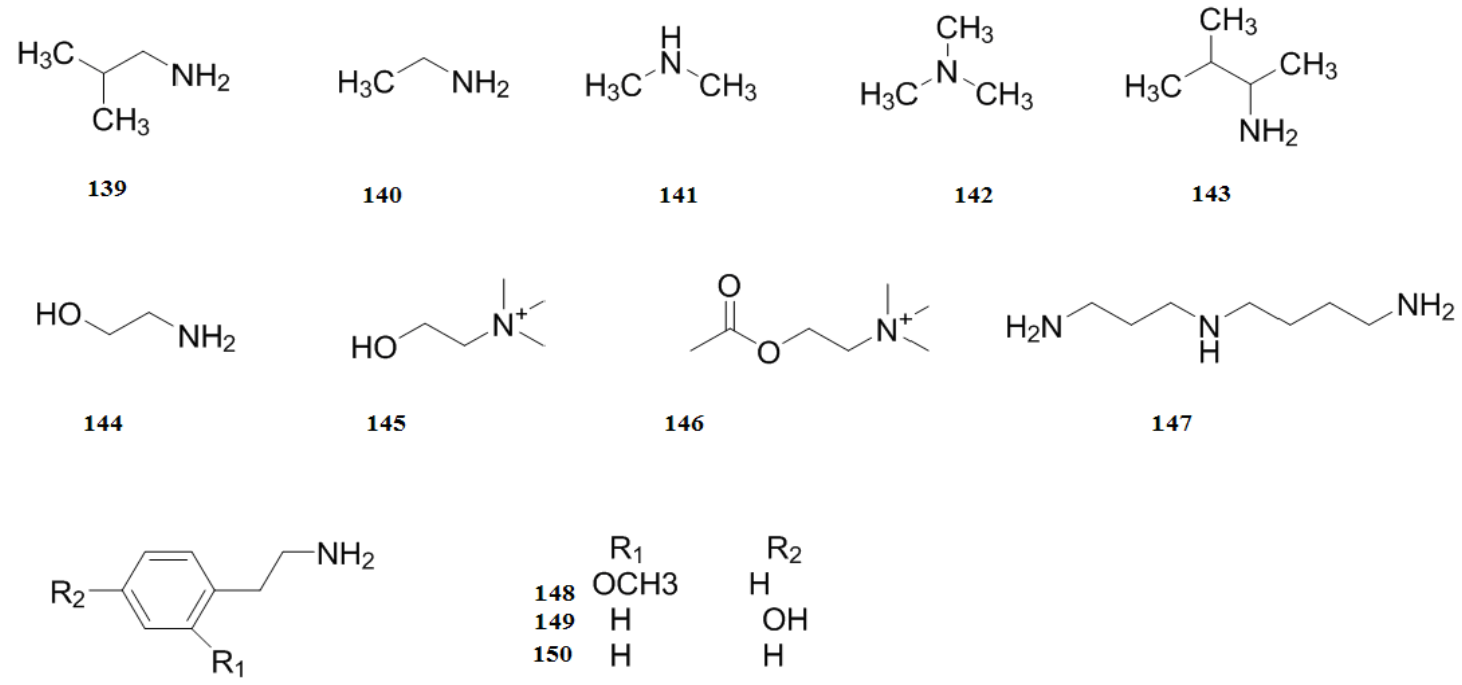

Figure 12. Chemical structures of other compounds in C. pinnatifida.

151

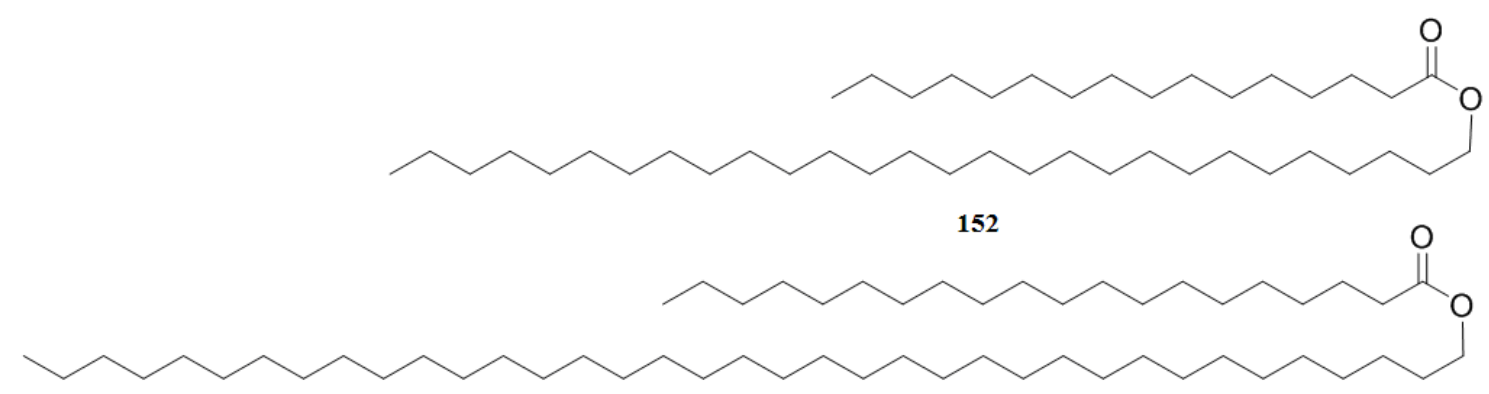

153
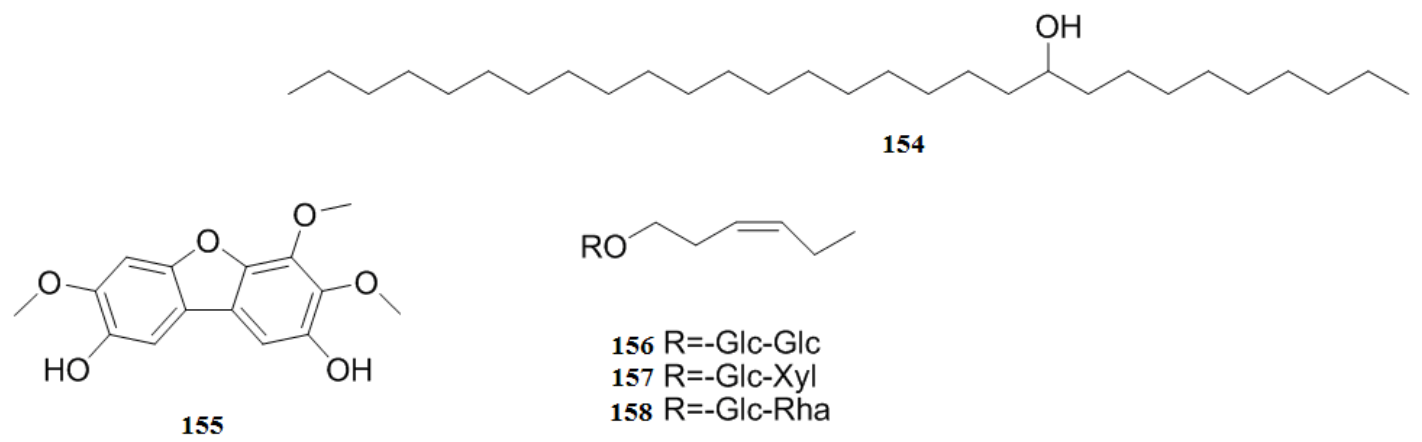


\section{Biological Properties}

\subsection{Cardiovascular System Effects}

\subsubsection{Lipid Regulating and Anti-atherosclerosis Effects}

Total flavonoids of the leaves from $C$. pinnatifida obviously decreased the serum levels of total cholesterol (TC) and triglyceride (TG) through gavage in high-fat/cholesterol rabbit models [50]. Total flavonoids of $C$. pinnatifida at a middle concentration of $50 \mu \mathrm{g} / \mathrm{mL}$ were able to promote the proliferation of preadipocytes but inhibit its differentiation. In addition, total flavonoids of C. pinnatifida inhibit mature adipocyte secretion of leptin and PAI-1 in a dose-dependent manner [51]. Moreover, total flavonoids of $C$. pinnatifida can markedly decrease the levels of total cholesterol (TC) and triglyceride (TG) in serum by controlling the gene expressions including FAS, HSL, TGH, SREBP-1c [52].

Atherosclerosis is a risk factor for coronary disease. There are a lot of theories about the pathogenesis of atherosclerosis, one of which is abnormal cholesterol levels [53]. The flavone extracted from C. pinnatifida by $70 \%$ ethanol obviously decreased the serum levels of total cholesterol (TC), triglyceride and low-density lipoprotein cholesterol (LDLC) in high-fat/cholesterol rabbit and rat models, suggesting its use to treat atherosclerosis [54].

Investigations have shown that the main antihyperlipidemic effect constituents of $C$. pinnatifida are hyperin and ursolic acid. Two animal models of hyperlipidemia were established in mice with $75 \%$ yolk and Triton-WR $1339400 \mathrm{mg} / \mathrm{kg}$ (ip), respectively. The animals were administrated with hyperoside or ursolic acid extracted from C. pinnatifida in two doses. The total cholestrol (TCH), triglyceride (TG), high density lipoprotein (HDL) and superoxide dismutase (SOD) activities in serum were measured. In comparison with control groups, TCH levels in all the dosed groups were significantly decreased, while HDL and SOD activity increased; the ratio of total cholesterol/high-density lipoprotein (TC/HDL) was reduced, too. This effect could lessen damages to vascular endothelium induced by oxygen free radical (OFR) in hyperlipoidemia, thus preventing atherosclerosis $[55,56]$. Total flavonoids of $C$. pinnatifida had significant antihyperlipidemic effects and enhanced the vascular function of hyperlipidemia model rats, the mechanism of which might be relevant to the increased levels of nitric oxide (NO) in the serum and the reduction in endothelin (ET) synthesis [57,58].

\subsubsection{Resistance to Chronic Heart Failure}

In many clinical trials, $C$. pinnatifida extract was confirmed to be effective in the treatment of patients with chronic heart failure defined as NYHA functional class II. There were no severe side effects observed [59-61]. In another clinical trial, C. pinnatifida extract WS 1442, a dry extract from hawthorn leaves with flowers (dry extract ratio $=4-6.6: 1$, extraction solvent: ethanol 45\%) standardized to $18.75 \%$ oligomeric procyanidines (OPC), was also effective in the treatment of patients with chronic stable New York Heart Association class-III heart failure. The most important constituents for the therapeutic effects of WS 1442 are OPC [62]. C. pinnatifida was capable of regulating and ameliorating cardiovascular system effects, as it enhanced myocardial contractility and expanded the coronary artery, lessened heart rhythm, myocardial oxygen consumption and peripheral 
resistance [63]. The mechanism of coronary artery expansion was relevant to the $\beta$-adrenergic receptor. The flavonoids of $C$. pinnatifida might be a new alternative botanical drug for chronic heart failure because of its good test results in pharmacological experiments.

\subsubsection{Antihypertensive Effects}

The extracts of $C$. pinnatifida could reduce blood pressure slowly and enduringly in mice, rabbits and cats, the mechanism of which was related to expanded peripheral vessels, and the active components were the flavanol dimers or multimers [64,65]. On compounding hypertension and hyperlipoidemia rats, extracts of $C$. pinnatifida at the doses of 1.5 and $2.25 \mathrm{~g} / \mathrm{kg} / \mathrm{d}$ could maintain rats' blood pressure [66].

\subsubsection{Anti-myocardial Ischemia and Reperfusion Injury Effect}

Total flavonoids isolated from the leaves of $C$. pinnatifida were able to reduce the degree of arrhythmia and lessen the burst size of LDH after damages in cardiocytes due to ischemia and hypoxia. Additionally, total flavonoids were able to enhance the endogenous oxygen purging system and reduce lipid peroxidation, showing it had an effect on relieving myocardial ischemia [67].

\subsection{Digestive System Effects}

\subsubsection{Gastrointestinal Function Regulating Effect}

The alcohol extract (extracted with 60\% alcohol) and the aqueous extract of $C$. pinnatifida had different effects on gastrointestinal function regulation. As for the alcohol extract, in the range of 2-8 $\mathrm{mg} / \mathrm{mL}$ (crude drugs), the alcohol extract of charred fruits of $C$. pinnatifida was able to significantly reduce the contractility of rat gastric and intestine smooth muscle strips in a dose-dependent manner. [68]. In another investigation, the alcohol extract of $C$. pinnatifida could significantly reduce the contractility of rat gastric and intestine smooth muscle strips in the range of 5-20 mg/mL (crude drugs) in a dose-dependent manner, and the extract at the dose of $20 \mathrm{mg} / \mathrm{mL}$ (crude drugs) could inhibit the stimulation induced by acetylcholine [69]. For the aqueous extract, in the range of 5-20 mg crude drugs $/ \mathrm{mL}$, the aqueous extract of $C$. pinnatifida significantly enhanced the contractility of rat gastric and intestine smooth muscle strips in a dose-dependent manner. The extract mentioned above at the dose of $20 \mathrm{mg} / \mathrm{mL}$ could enhance the intensive contraction induced by acetylcholine and antagonize the relaxation of intestinal smooth muscle induced by atropine [70].

A common side-effect of azithromycin is gastrointestinal effects, especially for children. In a study, when injected intravenously with azithromycin, if patients (children) took hawthron slices orally, the incidence of side-effects was lower compared with the control group $(p<0.05)$. This study demonstrated that $C$. pinnatifida was able to reduce the side-effects of azithromycin on the stomach and intestine without any reported additional side-effects [71]. 


\subsubsection{Digestive Enzyme Promotion Effects}

C. pinnatifida contains vitamin $\mathrm{C}$, vitamin $\mathrm{B} 2$, carotene and various organic acids, which could enhance the secretion of digestive enzymes and the enzyme activity within the stomach. Especially, amylase can enhance the activity of lipase which is able to directly help to digest fatty foods, and protease agonists from $C$. pinnatifida could enhance protease activity [72]. The organic acids were able to enhance mice gastrointestinal motility, and antagonize the relaxation of intestinal smooth muscle induced by atropine, though the organic acids had no effect on the stimulation of intestinal smooth muscles induced by neostigmine. This study demonstrated that it was a one-way regulation for intestinal motility [73].

\subsection{Effects on Pathogenic Microorganisms}

\subsubsection{Antibacterial Effects}

The antibacterial effects of $C$. pinnatifida have been comprehensively investigated. The juice squeezed from $C$. pinnatifida had antibacterial effects [74]. Additionally, the extracts of $C$. pinnatifida could inhibit various bacilli and cocci, such as Bacteroides forsythus, Song bacillus, Smith bacillus, Proteusbacillus vulgaris, Bacillus anthraci, Corynebacterium diphtheria, Typhoid bacillus and Streptococcus hemolyticus. Skin disinfectants with extract of $C$. pinnatifida fruit pit as the main germicidal ingredient had preferable sterilizing effect and stability on Escherichia coli, Staphylococcus aureus, Pseudomonas aeruginosa and Candida albicans [75].

\subsubsection{Synergistic Antibacterial Effects}

In vitro, the minimal inhibitory concentrations (MICs) of oxacillin, ampicillin sodium plus sulbactam sodium, ampicillin, cephazolin, and active components of C. pinnatifida were 256, 512, 512,128 , and $1,024 \mu \mathrm{g} / \mathrm{mL}$ against methicillin-resistant staphylococcus aureus (MRSA), respectively. However, when combined with the active components of $C$. pinnatifida at its sub-MIC $(128 \mu \mathrm{g} / \mathrm{mL})$ concentration, the MICs of the above four $\beta$-lactam antibiotics were 2, 32, 16 and $2 \mu \mathrm{g} / \mathrm{mL}$ respectively. The results demonstrated that these active components of $C$. pinnatifida had a synergistic antibacterial effect on MRSA when combined with $\beta$-lactam antibiotics [76].

In an experiment, the bacterial strains were the standard MRSA strain WHO-2 (WHO-2) and 45 clinical MRSA strains. WHO-2 possessed a high level of resistance to oxacillin (MIC $=512 \mathrm{mg} / \mathrm{L}$ ) and harbors the mecA gene. The 45 clinical strains were all resistant to oxacillin (MIC $>4 \mathrm{mg} / \mathrm{L}$ ) and harbor the mecA gene. (+)-Catechin (C), (-)-epicatechin gallate (ECg) and (-)-epigallocatechin (EGC) were isolated from fructus crataegi (hawthorn) guided by antibacterial sensitization activity. The combination of $(+)$-catechin (C) and (-)-epicatechin gallate $(\mathrm{ECg})$ could enhance the activity of $\beta$-lactam antibiotics against MRSA in vitro and in vivo, which might be related to the increased accumulation of antibiotics within MRSA via suppression of important efflux pump gene expression. It was demonstrated by two-fold dilution and checkerboard methods that $\mathrm{C}(128 \mathrm{mg} / \mathrm{L})$ combined with $\mathrm{ECg}(16 \mathrm{mg} / \mathrm{L})$ had the greatest effect, and the combination also reduced the bacterial load in blood of septic mice challenged with a sub-lethal dose of MRSA. The mechanism is related to increased daunomycin accumulation within MRSA and down-regulated the mRNA expression of norA, norC and abcA, three important efflux pumps of MRSA [77]. 


\subsubsection{Antiviral Effects}

Human immunodeficiency virus (HIV) releases itself from an HIV-infected cell using serine protease, followed by attack on other cells. C. pinnatifida was found to inhibit the activity of serine protease, followed by decreasing diffusion rate of HIV in vivo [78]. Maslinic acid isolated from C. pinnatifida had a prominent effect on inhibiting the activity of HIV-1 protease. When the concentration was $17.9 \mathrm{mg} / \mathrm{mL}$, the inhibitive rate is $100 \%$ [79]. Therefore, maslinic acid is considered as a potential candidate for novel anti-HIV therapeutics.

\subsection{Effects on Tumors and the Immune System}

\subsubsection{Anticancer and Sperm Distortion Inhibiting Effects}

The anticancer effect of maslinic acid was investigated in 1989. Maslinic acid isolated from C. pinnatifida exhibited cytotoxicity on P-38 cancer cells $\left(\mathrm{ED}_{50}=13.0 \mu \mathrm{g} / \mathrm{mL}\right)$ [80]. Total flavonoids isolated from $C$. pinnatifida had no effect on normal cells, but could obviously enhance calcium concentration in tumor cells. In vitro, total flavonoids inhibited and killed Hep-2 tumor cells by calcium overload, as well as inhibited DNA biosynthesis of tumor cells [81]. Aqueous extracts of C. pinnatifida were found to inhibit sperm distortion of mice induced by cyclophosphamide, which obviously reduced the number of distorted sperm. The mechanism was related to the abundant linoleic acid and vitamin $\mathrm{C}$ in C. pinnatifida [82].

\subsubsection{Immunoregulating Effects}

In an experiment, the $100 \%$ decoction of $C$. pinnatifida was used in mice (p.o., $0.2 \mathrm{~mL} / 10 \mathrm{mg} / \mathrm{d}$, for 9 days). It was shown that the decoction could increase the weight of the immune organs of the mice (thymus and spleen), raise T lymphocyte transformation rate and T lymphocyte ANAE cell percentage, indicating the decoctum of $C$. pinnatifida has an obvious improving effect on the cellular immune function of the mice, which also provides an experimental basis for the clinical application [83]. The injection of $C$. pinnatifida (water extract and alcohol precipitate, $1 \mathrm{~g}$ crude drugs $/ \mathrm{mL}$ ) had the same effect as the decoction [84]. Sitosterol isolated from $C$. pinnatifida was able to significantly increase the leucocyte count and enhance the phagocytic activity of macrophages, and had effects on spleen and lymphocytes in mice model of immunosuppression induced by cyclophosphamide (CTX) [85]. It was confirmed that the polysaccharides extracted from $C$. pinnatifida were able to enhance the spleen, thymus and the phagocytic activity of macrophages, promote the formation of hemolysin and hemolysis plaque of mice [86].

\subsection{Endocrine System Effects}

Endocrine system imbalance is a major factor of diabetes. The regulation of the hepatic glucose output through glycogenolysis is an important target for type II diabetes therapy. Glycogenolysis is catalyzed in liver, muscle and brain by tissue specific isoforms of glycogen phosphorylase (GP). Because of its central role in glycogen metabolism, GP had been exploited as a model for structure assisted design of potent inhibitors, which might be relevant to the control of blood glucose 
concentrations in type II diabetes [87,88]. Maslinic acid isolated from $C$. pinnatifida was found to inhibit GP in moderate strength; the IC50 was $28 \mu \mathrm{mol} / \mathrm{L}$. As glycogen phosphorylase inhibitors, the best effect of maslinic acid derivatives was 4 times better than maslinic acid [89]. Maslinic acid (10 $\mu \mathrm{g} / \mathrm{kg} / \mathrm{day}$ or $30 \mu \mathrm{g} / \mathrm{kg} /$ day, two weeks) was found to obviously lessen the levels of blood glucose in KK-Ay mice by lessening insulin resistance of KK-Ay mice, these results suggested that maslinic acid might be investigatedas a new drug for type II diabetes treatment [90].

\subsection{Coagulation System Effects}

In vitro, the extracts from the leaves of $C$. pinnatifida were reported to inhibit platelet aggregation of rabbit [91]. In another study, the IC50 of $C$. pinnatifida on platelet aggregation induced by ADP was $1.388 \%$ (g crude drugs per $100 \mathrm{~mL}$ ) [92]. In acute blood stasis rat model, total flavonoids obviously influenced the hemorheology, which decreased viscosity of plasma and hematocrit [93]. Total flavonoids isolated from the leaves of $C$. pinnatifida were found to inhibit thrombogenesis caused by vascular endothelial injury of artery. The mechanism is related to the enhancement of the surface charge and speeding up the fluxion of erythrocyte and soterocyte, lessening the gather and adhesion [94,95]. At low doses (between 100-500 mg/kg), C. pinnatifida water extracts was reported to inhibit platelet function significantly in Wistar albino rats. The extracts were able to change the bleeding time and the closure time, which determined by the PFA-100 and thromboxane B2 levels [96].

\subsection{Other Effects}

\subsubsection{Antiinflammatory Effects}

The inhibitory effect of ethanol extract from the leaves of $C$. pinnatifida on mice ear inflammation induced by dimethylbenzene was investigated [97,98]. The results showed ethanol extract of $C$. pinnatifida has definite antiinflammatory effects.

\subsubsection{Antioxidant Effects}

Total flavonoids of $C$. pinnatifida leaves was found to have a strong ability to scavenge oxygen free radicals, enhance superoxide dismutase (SOD) activities, and lessen malondialdehyde (MDA) levels. This result demonstrated that total flavonoids of $C$. pinnatifida leaves had good effects on protecting brain tissue, nephridial tissue, hepatic tissue and neuron by remitting oxidative stress [99-102].

\subsubsection{Osteoporosis Inhibiting Effects}

In a simulative animal model of osteoporosis induced by menopause, maslinic acid isolated from C. pinnatifida was found to inhibit osteoporosis. The mechanism was that maslinic acid was able to inhibit downstream-signal (NFאB) activities and transcription-factor (NFATcl) expressions, but it had no effects on transcriptional activities of NFATcl. Additionally, maslinic acid could also regulate the downstream-signalling (MAPK); but it had no effects on calcium flow oscillation. Therefore, the results suggested maslinic acid might be used as a new drug against osteoporosis induced by menopause [103]. 


\subsubsection{Retina Protecting Effects}

In an experiment, experimental rabbits were contaminated by inhaling $\mathrm{CS}_{2}$ for 3 continuous hours on 6 consecutive days a week for a total of 3 weeks. The rabbits in the treatment group were given "haw drink compound" (water decoction with Crataegus pinnatifida, Lycium barbarum, Fructus jujubae) before contamination. After 3 weeks of the experiment, the results showed that the ultrastructures of the retinal tissues of the control group were more abnormal than those of the treatment group and the normal group. Every layer cell of the retinal in the control group showed apparent degenerative changes, but that in the treatment group was normal. This investigation demonstrated that haw drink compound could improve the tolerance to $\mathrm{CS}_{2}$ toxicity in inducing the retinal damage of rabbits [104].

\section{Toxicology}

C. pinnatifida has been used for hundreds of years as an important traditional herbal medicine in China, as well as a daily foodstuff. However, studies of the relative systematic toxicity and safety of C. pinnatifida are lacking. So far, oral corn pollen haw liquor (an oral solution containing corn, pollen and haw) was demonstrated no have no genotoxicity effects [5]. Additionally, in order to ensure the safety of drug use, acute and long-term toxicity experiments with "semen cassiae hawthorn oat" (a capsule contains semen cassia, hawthorn and oat) were investigated. For the acute toxicity reactions, the semen cassiae hawthorn oat was orally given one-time at $10 \mathrm{~g} / \mathrm{kg}$ dosages (the maximum tolerated dose in mice), and observed continuously for 14 days. For the long-term toxicity reactions, the semen cassiae hawthorn oat was orally given continuously for 8 weeks at low $(1.6 \mathrm{~g} / \mathrm{kg})$ and high $(2 \mathrm{~g} / \mathrm{kg})$ dosages. In the acute toxicity experiments, there were no toxic reactions or animal deaths. In the long-term toxicity experiments, there was no significant differences in the general state, weight changes, hematological indexes, biochemical indexes and organ coefficients in rats of low and high dosage group when compared with the control group. Pathologic examination did not show any structural and cellular abnormalities of each organ [105]. The above results demonstrated that C. pinnatifida is safe and non-toxic for experimental animals.

\section{Future Perspectives and Conclusions}

Medicinal plants are universally considered as important sources of new chemical substances with potential therapeutic effects. C. pinnatifida has long been used in Traditional Chinese Medicine for the treatment of cardiovascular disease, dyspepsia, infections and cancers. The flavonoids are considered to be the major bioactive constituents. C. pinnatifida has been of increasing interest in recent years, and many traditional uses have been investigated, but there is not enough systemic data about the toxicity and safety of $C$. pinnatifida, and few target-organ toxicity evaluations have been documented. Therefore, more investigations should be done regarding the toxicity and pharmacokinetics of $C$. pinnatifida.

In Traditional Chinese Medicine, $C$. pinnatifida is commonly used in compositions with other herbs and not used alone. Although many of the experimental results validate that $C$. pinnatifida exhibits significant pharmacological effects when used alone, it's important to investigate the pharmacological effects and molecular mechanisms of $C$. pinnatifida combined with other herbs based on modern concepts of disease pathophysiology. Furthermore, drug target-guided and bioactivity-guided isolation 
and purification of the chemical constituents and subsequent evaluation of the pharmacologic effects will promote the development of bioactive constituents and expand our knowledge of $C$. pinnatifida. Detailed investigations of the pharmacology, molecular mechanisms of action and systems biology will help to ensure which chemical constituents or multiple ingredients contribute to its pharmacological effects and help develop new effective drugs which could produce enormous benefit to society and the economy.

\section{Conflicts of Interest}

The authors declare no conflict of interest.

\section{References}

1. Gao, G.Y.; Feng, Y.X. The literatures of traditional Chinese medicine hawthorn. Zhongguo Zhong Yao Za Zhi 1994, 5, 259-260.

2. Wang, C.L.; Lu, B.Z.; Hou, G.L. Chemical constituent, pharmacological effects and clinical application of Crataegus pinnatifida. Strait Pharm. J. 2010, 3, 75-78.

3. Wu, S.J.; Li, Q.J.; Xiao, X.F.; Li, M.; Yang, X.R.; Lv, T. The research of chemical constituent and pharmacological effects of Crataegus pinnatifida. Drug Eval. Res. 2010, 4, 316-319.

4. Editorial Committee of Dictionary of Chinese Materia Medica. Dictionary of Chinese Materia Medica, 2001, ed.; People's Medical Publishing House: Beijing, China, 2001.

5. Yang, B.S.; Li, Y.L.; Niu, L.Y.; Feng, H.G.; Li, W.L. Experimental study on genotoxicity of oral corn pollen haw liquor. J. Xinxiang Med. Coll. 1998, 1, 6-8.

6. Melikoglu, G.; Mericli, F.; Mericli, A.H. Flavonoids of Crataegus orientalis. Boll. Chim. Farm. 1999, 7, 351-352.

7. Titova, A.A.; Batyuk, V.S. C-glycosides of crataegus curvisepala. Chem. Nat. Comp. 1986, 22, 348.

8. Nikolov, N.; Batyuk, V.S.; Kovalev, I.P.; Ivanov, V. Glycoflavonoids of crataegus monogyna and C. pentagyna. Chem. Nat. Compd. 1973, 9, 110.

9. Bykov, V.I.; Glyzin, V.I.; Ban'kovskii, A.I. Flavonoids of the genus crataeugs. The structure of bioquercetin. Chem. Nat. Compd. 1972, 8, 657.

10. Nikolov, N. New flavone c-biosides from Crataegus monogyna and Cr. Pentagyna. Chem. Nat. Compd. 1975, 11, 434-435.

11. Batyuk, V.S.; Prokopenko, A.P.; Kolesnikov, D.G. New flavonoids from the leaves of Crataegus curvisepala lindm. Chem. Nat. Compd. 1965, 1, 225-226.

12. Zhang, P.C.; Xu, S.X. Flavonoid ketohexosefuranosides from the leaves of Crataegus pinnatifida Bge.var.major N.E.Br. Phytochemistry 2001, 57, 1249-1253.

13. El-Mousallamy, A.M.D. Chemical investigation of the constitutive flavonoid glycosides of the leaves of Crataegus sinaica. Nat. Prod. Sci. 1998, 4, 53-57.

14. Nikolov, N.; Dellamonica, G.; Chopin, J. Di-c-glycosyl flavones from Crataegus monogyna. Phytochemistry 1981, 20, 2780-2781.

15. Batyuk, V.S.; Chernobrovaya, N.V.; Prokopenko, A.P. Cratenacin-A new flavone glycoside from Crataegus curvisepala. Chem. Nat. Compd. 1966, 2, 90-93. 
16. Kashnikova, M.V. Flavonoids of the flowers of Crataegus sanguinea. Chem. Nat. Compd. 1984, 20, 105-106.

17. Sözer, U.; Dönmez, A.A.; Mericli, A.H. Constituents from the leaves of Crataegus davisii Browicz. Sci. Pharm. 2006, 74, 203-208.

18. Zhang, P.C.; Xu, S.X. Chamical constituents from the leaves of Rataegus pinnatifida Bge. Var. Major N.E.Br. Yao Xue Xue Bao 2001, 10, 754-757.

19. Mericli, A.H.; Ergezen, K. Flavonoids of Crataegus tanacetifolia (Rosaceae), an endemic species from Turkey. Sci. Pharm. 1994, 3, 277-281.

20. Bykov, V.I.; Glyzin, V.I.; Ban'kovakii, A.I. Pinnatifidin-a new flavonol glycoside from Crataegus pinnatifida. Chem. Nat. Compd. 1972, 8, 699-701.

21. Zhao, Y.P.; Wang, C.X.; Du, L.X. Study on chemical constituents in fruits and leaves of Crataegus L. Beverage Ind. 2002, 6, 8-12.

22. Kowalewski, Z.; Mrugasiewicz, K. New falvanone glycosides in Crataegus phaenopyeum. Planta Med. 1971, 19, 311-317.

23. Shahat, A.A.; Ismail, S.I.; Hammouda, F.M.; Azzam, A.A.; Lemiere, G.; de-Bruyne, T.; de-Swaef, S.; Pieters, L.; Vlietinck, A. Anti-HIV activity of flavonoids and proanthocyanidins from Crataegus sinaica. Phytomedicine 1998, 2, 133-136.

24. Nikolov, N.; Batyuk, V.S.; Ivanov, V. Crateside-A new flavonol glycoside from Crataegus monogyna and C. pentagyna. Chem Nat Comp 1973, 9, 150-151.

25. Petrova, V.P. Catechins and leuconathocyanins of Crataegus fruit. Ukr. Bot. Zh. 1972, 2, 144-147.

26. Svedström, U.; Vuorela, H.; Kostiainen, R.; Tuominen, J.; Kokkonen, J.; Rauha, J.P.; Laakso, I.; Hiltunen, R. Isolation and identification of oligomeric procyanidins from Crataegus leaves and flowers. Phytochemistry 2002, 60, 821-825.

27. Shi, Y.P.; Ding, X.B. Studies on chemical constituents from fruit of Crataegus pinnatifida. Chin. Tradit. Herbal Drugs 2000, 3, 173-175.

28. Song, S.J.; Chen, J.; Kou, X.; Song, Y.H.; Xu, S.X. Chemical constituents from leaves of Crataegus pinnatifida Bge (I). J. Shenyang Pharm. Univ. 2006, 23, 88-90.

29. Chen, L.S.; Lv, L.; Xu, S.W.; Xin, Y. Study on the Triterpene Acids in Fruit of Crataegus pinnatifida. Lishizhen Med. Mater. Med. Res. 2008, 19, 2909-2910.

30. Ikeda, T.; Ogawa, Y.; Nohara, T. A new triterpenoid from Crataegus cuneata. Chem. Pharm. Bull. 1999, 10, 1487-1488.

31. García, M.D.; Sáenz, M.T.; Ahumada, M.C. A.Cert.Isolation of three triterpenes and several aliphatic alcohols from Crataegus monogyna Jacq. J. Chromatogr. A 1997, 767, 340-342.

32. Sun, X.F.; Yao, Q.Y. Chemical constituents from seed of Crataegus pinnatifida Bge. Chin. Tradit. Herbal Drugs 1987, 18, 441-454.

33. Huang, X.X.; Niu, C.; Gao, P.Y.; Li, L.Z.; Ming, M.; Song, S.J. Chemical constituents from the leaves of the Crataegus pinnatifida Bge (III). J. Shenyang Pharm. Univ. 2010, 27, 615-618.

34. Zhang, P.C.; Xu, S.X.; Guo, H. Study on chemical component in the fruit of Crataegus pinnatifida. J. Shenyang Inst. Chem. Technol. 1999, 16, 87-89.

35. Song, S.J.; Li, L.Z.; Gao, P.Y.; Peng, Y.; Yang, J.Y.; Wu, C.F. Terpenoids and hexenes from the leaves of Crataegus pinnatifida. Food Chem. 2011, 129, 933-939. 
36. Gao, P.Y.; Li, L.Z.; Peng, Y.; Li, F.F.; Niu, C.; Huang, X.X.; Ming, M.; Song, S.J. Monoterpene and lignin glycosides in the leaves of Crataegus pinnatifida. Biochem. Syst. Ecol. 2010, 38, 988-992.

37. Ahmed, A.A.; Khattab, A.M.; Grace, M.H.; Sahl, M.M. A new eudesmanolide from Crataegus flava fruits. Fitoterapia 2001, 72, 759-759.

38. Schrall, R.; Becker, H. Production of catechins and oligomeric proanthocyanidins in callus and suspension cultures of Crataegus monogyna, C. oxyacantha and Ginkgo biloba. Planta Med. 1977, 4, 297-318.

39. Hao, D.F.; Yang, R.P.; Zhou, Y.Z.; Chen, H.; Li, Z.F.; Pei, Y.H. Chemical constituents from the leaves of the Crataegus pinnatifida Bge (II). J. Shenyang Pharm. Univ. 2009, 26, 282-284.

40. Chen, J.; Song, S.J.; He, J.; Xu, S.X. A new biphenyl glucoside from the leaves of Crataegus pinnatifida. J. Shenyang Pharma. Univ. 2006, 23, 430-431.

41. Huang, X.X.; Zhou, C.C.; Li, L.Z.; Peng, Y.; Lou, L.L.; Liu, S.; Li, D.M.; Ikejima, T.; Song, S.J. Cytotoxic and antioxidant dihydrobenzofuran neolignans from the seeds of Crataegus pinnatifida. Fitoterapia 2013, 91, 217-223.

42. Huang, X.X.; Zhou, C.C.; Li, L.Z.; Li, F.F.; Lou, L.L.; Li, D.M.; Ikejima, T.; Peng, Y.; Song, S.J. The cytotoxicity of $8-O-4^{\prime}$ neolignans from the seeds of Crataegus pinnatifida. Bioorg. Med. Chem. Lett. 2013, 23, 5599-5604.

43. Wang, X.S.; Che, Q.M.; Li, Y.M.; He, Y.Q. A study on chemical constituents in seeds of Crataegus pinnatifida Bge.var. Major N. E. Br. Zhongguo Zhong Yao Za Zhi 1999, 17, 739-740.

44. Chapman, G.W., Jr.; Horvat, R.J.; Payne, J.A. The nonvolatile acid and suger composition of mayhaw fruit. Food Qual. 1991, 14, 435-439.

45. Roddewig, C.; Hensel, H. Reaction of local myocardial blood flow in non-anesthetized dogs and anesthetized cats to the oral and parenteral administration of a Crateagus fraction (Oligomere procyanidines). Arzneim.-Forsch. 1977, 27, 1407-1410.

46. Evdokimova, O.V.; Samylina, I.A.; Nesterova, O.V. Examination of a lipophilic Crataegus fruit fraction. Farmatsiya 1992, 41, 60-61.

47. Hobbs, S.; Foster, S. Hawthom: a literature review. Herbal Gram. 1990, 22, 19-33.

48. Kokubun, T.; Harborne, J.B.; Eagles, J.; Waterman, P.G. Dibenzofuran phytoalexins from the sapwood tissue of Photinia, Pyracantha and Crataegus species. Phytochemistry 1995, 5, 1033-1037.

49. Jennifer E. Edwards, J.E.; Brown, P.N.; Talent, N.; Dickinson, T.N.; Shipley, P.R. A review of the chemistry of the genus Crataegus. Phytochemistry 2012, 79, 5-26.

50. Liu, Q.L.; Yang, Z.L. Comparison of total flavonoids of different purities from Folium crataegi in effect of antihyperlipidemia. Strait Pharm. J. 2008, 20, 32-52.

51. Liu, X.Y.; Zhou, L.; Liang, R.Y. Study on lipid regulation mechanism of total flavonoids from Folium crataegi by 3T3-L1 cells. Chin. Arch. Tradit. Chin. Med. 2009, 27, 1066-1068.

52. Xie, W.H.; Sun, C.; Liu, S.M. Effect of hawthorn flavanone on blood-fat and expression of lipogenesis and lipolysis genes of hyperlipoidemia model mouse. Zhongguo Zhong Yao Za Zhi 2009, 34, 224-229.

53. Yang, R.M.; Chen, H.M.; Gao, N.N.; Song, X.; Li, J.L.; Cai, D.Y. Mechanism of early atherosclerosis in guinea pig: abnormal metabolism of LDL-C. Acta Lab. Anim. Sci. Sin. 2011, 23, 237-241.

54. Liu, J.; Sun, H.B.; Duan, W.G.; Mu, D.Y.; Zhang, L.Y. Maslinic acid reduces blood glucose in KK-Ay mice. Biol. Pharm. Bull. 2007, 30, 2075-2078. 
55. Li, G.H.; Sun, J.Y.; Zhang, X.L. Experimental studies on antihyperlipidemia effects of two compositions from hawthorn in mice. Chin. Tradit. Herbal Drugs 2002, 33, 50-52.

56. Yang, Y.J.; Lin, J.; Wang, C.M. Effect of FHL on intervening early hyperlipoidemia model rat. Chin. Tradit. Herbal Drugs 2008, 39, 1848-1850.

57. Ye, X.Y.; Zhang, L.; Shen, J. Effect of hawthorn leaf flavonoids on metabolism of glucose and lipids in diabetic mice. Chin. Tradit. Herbal Drugs 2005, 36, 1683-1686.

58. Yang, Y.J.; Wang, C.M.; Dang, X.W. Protection of flavonoids in hawthorn leaf against vascular disfunction of hyperlipoidemic rats. Chin. Tradit. Herbal Drugs 2007, 38, 1687-1690.

59. Degenring, F.H.; Suter, A.; Weber, M.; Saller, R. A randomised double blind placebo controlled clinical trial of a standardised extract of fresh Crataegus berries $\left(\right.$ Crataegisan $\left.{ }^{\circledR}\right)$ in the treatment of patients with congestive heart failure NYHA II. Phytomedicine 2003, 11, 363-369.

60. Schmidt, U.; Kuhn, U.; Ploch, M.; Hübner, W.-D. Efficacy of the hawthorn (Crataegus) preparation LI 132 in 78 patients with chronic congestive heart failure defined as NYHA functional class II. Phytomedicine 1994, 1, 17-24.

61. Zapfe, G. Clinical efficacy of Crataegus extract WS ${ }^{\circledR} 1442$ in congestive heart failure NYHA class II. Phytomedicine 2001, 49, 262-266.

62. Tauchert, M. Efficacy and safety of Crataegus extract WS 1442 in comparison with placebo in patients with chronic stable New York Heart Association class-III heart failure. Am. Heart J. 2002, 155, 910-915.

63. Ju, X.Y.; Fang, T.H.; Zhang, W.T. Effect of freezedrying powder of total flavonoids in chinese hawthorn leaf on myocardial infarction caused by coronary ligation of anesthetic dogs. J. Nanjing Univ. Tradit. Chin. Med. 2005, 19, 381-383.

64. Editorial Committee of Modern Clinical Chinese Materia Medica. Modern Clinical Chinese Materia Medica, 1998 ed.; China Press of Traditional Chinese Medicine: Beijing, China, 1998.

65. Yang, X.P. Medicinal value of Crataegus pinnatifida. Jilin Med. J. 1998, 19, 41.

66. Yuan, Y.; Zhao, J.; Gao, H.J.; Wang, J.H. Experimental study on effect of hawthorn on compounding hypertension and hyperlipoidemia rats. J. Xinjiang Med. Univ. 2013, 35, 52-27.

67. Yu, B.; Li, H.Y.; Zhang, L. Protective effect of total flavonoids from hawthorn leaf on ligation-induced acute myocardial ischemia in anesthetized dogs. Tradit. Chin. Drug Res. Clin. Pharmacol. 2008, 19, 461-464.

68. Huang, S.S.; Lin, Y.; Diao, Y.P. Effects of charred fructus Crataegi alcohol extract on contractility of isolated rat gastric and intestine muscle strips. Prog. Mod. Biomed. 2009, 9, 612-614.

69. Deng, S.; Lin, Y.; Diao, Y.P. Effects of hawthorn alcohol extract on the contractility of isolated rat gastric and intestine muscle strips. Prog. Mod. Biomed. 2009, 9, 1262-1264.

70. Wen, X.P.; Deng, S.; Lin, Y.; Diao, Y.P.; Huang, S.S.; Zhang, H.L. Effects of Folium crataegi water extract on the contractility of isolated gastric and intestinal muscle strips in rats. China Pharm. 2010, 21, 978-980.

71. Yang, S.X.; Wang, X.C.; Wang, Z.X.; Han, J.; Liu, D.L.; Zhang, Y. Discussion of Haw foods in the reduction of azithromycin's side-effect. Chin. Med. Herald 2010, 7, 175-176.

72. Editorial Committee of Pharmacology of Chinese Materia Medica. Pharmacology of Chinese Materia Medica, 2000 ed.; People’s Medical Publishing House: Beijing, China, 2000. 
73. Wu, J.H.; Sun, J.Y. Effect of Crataegus pinnatifida organic acids on the gastrointestinal motility. Shanxi J. Tradit. Chin. Med. 2009, 25, 1402-1403.

74. Lin, L.; Chen Y.J.; Li, L.; Cao, Y.; Sun, Q.X. Experimental observation on germicidal efficacy of hawthorn liquid and its influencing factors. Chin. J. Disinfect. 2000, 17, 85-88.

75. Li, C.Q.; Wu, W.; Tong, Y. Study on germicidal efficacy of extract of hawthorn fruit pit and its influencing factors. Chin. J. Disinfect. 2007, 24, 50-52.

76. Jiang, K.; Qin, R.X.; Zhu, T.F.; Zhou, H.; Zheng, J. Antibacterial activity of active component of Crataegus pinnatifida combined with $\beta$-lactam antibiotics against methicillin-resistant Staphylococcus aureus in vitro. Acta Acad. Med. Milit. Tert. 2009, 19, 1887-1889.

77. Qin, R.X.; Xiao, K.K.; Li, B.; Jiang, W.W.; Peng, W.; Zheng, J.; Zhou, H. The combination of Catechin and Epicatechin Gallate from Fructus crataegi potentiates $\beta$-Lactam antibiotics against Methicillin-resistant Staphylococcus aureus (MRSA) in vitroand in vivo. Int. J. Mol. Sci. 2013, 14, 1802-1821.

78. Wang, B.; Qiu, W.W.; Yu, Y.Y.; Yang, F.; Tang, J. Progress of study on maslinic acid. Chin. Bull. Life Sci. 2009, 21, 264-269.

79. Xu, H.X.; Zeng, F.Q.; Wan, M.; Sim, K.Y. Anti-HIV triterpene acids from Geum japonicum. J. Nat. Prod. 1996, 59, 643-645.

80. Numata, A.; Yang, P.; Takahashi, C.; Fujiki, R.; Nabae, M.; Fujita, E. Cytotoxic triterpenes from a Chinese medicine, goreishi. Chem. Pharm. Bul1. 1989, 37, 648-651.

81. Zhang, Y.; Li, H.W.; Sun, J.P. Extraction-Separation of total flavone from the fruit of Crataegus pinnatifida and anticancer activities in vitro. Chin. Tradit. Herbal Drugs 2004, 35, 787-789.

82. Cui, T.C.; Liu, X.Q.; Xu, H.Q.; Wu, G.J.; Zhang, Z.B. Effect of the extracts of C. pinnatifida on inhibiting sperm distortion of mice induced by cyclophosphamide. Chin. Public Health 2002, 3, 266-267.

83. Chang, J.; Jin, Z.P.; Gao, G. Effect of decoctum of Crataegus pinnatifida Bge on the cellular immune function of mice. J. Baotou Med. Coll. 1996, 12, 10.

84. Jin, Z.C.; Bai, L.H. Effect of hawthorn injection on the immunity of rabbit. Chin. Tradit. Herbal Drugs 1992, 23, 592-593.

85. Dong, H.; Zhang, T.P.; Peng, S.M.; Li, J.; Zhang, H.Y. Extraction of sitosterol from hawthorn fruits and effect of sitosterol on immunological function and serum lipid. Nat. Prod. Res. Dev. 2009, 21, 60-63.

86. Yan, Q.G. Effect of hawthorn polysaccharides on immunity of mice. J. China Tradit. Chin. Med. Inf. 2009, 29, 134-135.

87. Oikonomakos, N.G. Glycogen phosphorylase as a moleculartarget for type II diabetes therapy. Curr. Protein Pept. Sci. 2002, 3, 561-586.

88. Somsák, L.; Nagya, V.; Hadady, Z.; Docsa, T.; Gergely, P. Glucose analog inhibitors of glycogen phosphorylases as potential antidiabetic agents: Recent developments. Curr. Pharm. Des. 2003, $15,1177-1189$.

89. Wen, X.A.; Zhang, P.; Liu, J.; Zhang, L.Y.; Wu, X.M.; Ni, P.Z.; Sun, H.B. Pentacyclic triterpenes. Part 2: Synthesis and biological evaluation of maslinic acid derivatives as glycogen phosphorylase inhibitors. Bioorg. Med. Chem. Lett. 2006, 16, 722-726. 
90. Liu, B.L.; Dong, J.S.; Ni, X.H. In vivo study on flavonoids extraction from hawthorn fruits and effects of hawthorn leaves flavonoids. Food Sci. 2007, 28, 324-327.

91. Yang, L.P.; Wang, C.L.; Wang, Y.L.; Li, Y.S.; Fu, S.X. The effects of extracts from the leaves of Crataegus pinnatifida on platelet aggregation of rabbits and myocardial ischemia of rats. Chin. Tradit. Herbal Drugs 1993, 24, 482-483.

92. Shi, J.; Wang, Z.X.; Lu, X.H. Synergetic Antiplatelet effects of Fructus crataegi and Rhizoma alismatis. Chin. Tradit. Herbal Drugs 1996, 27, 350-352.

93. Jiang, K.Y.; Gu, Z.L.; Ruan, C.G. Effect of WH505 on experimental thrombosis and its mechanism. Chin. Tradit. Patent Med. 2000, 20, 203-205.

94. Jiao, L.P. Effect of flavone mixture of crataegus leaves on hemorheology index of acute blood stasis rat model. Chin. Remedies Clin. 2008, 8, 49-50.

95. Yang, Y.J.; Dong, X.Q.; Guo, J.J. Experimental research of blood-quickening stasis-transforming actions of hawthorn leaf flavonoids. Hebei Med. 2009, 31, 22-23.

96. Shatoor, A.S.; Soliman, H.; Al-Hashem, F.; El-Gamal, B.; Othman, A.; El-Menshawy, N. Effect of hawthorn (Crataegus aronia syn. Azarolus (L)) on platelet function in albino wistar rats. Thrombosis Res. 2012, 130, 75-80.

97. Quan, Y.C.; Guan, L.P. Anti-inflammation,analgesic effects of extract of hawthorn leaf. Lishizhen Med. Mater. Med. Res. 2006, 17, 556-557.

98. Wang, Y.; Sun, G.H.; Zhang, R.F.; Zhang, M.; Wang, L.J. Anti-inflammation, analgesic effects of extracts of leaves of Crataegus pinnatifida. Acta Chin. Med. Pharmacol. 2012, 40, 38-39.

99. Ji, Y.S.; Li, H.; Yang, S.J. Protective effect and its molecular mechanism of FMCL on PC 12 cells apoptosis induced by H2O2. Chin. Pharmacol. Bull. 2006, 22, 760-762.

100. Li, L.; Lv, H.; Pang, H. Anti-aging effect of total flavone of hawthorn leaf. Lishizhen Med. Mater. Med. Res. 2007, 9, 2143-2144.

101. Chen, Z.Y.; Yan, M.X.; He, B.H. The Change and the immpact of IFHL on oxidative stress in the formation of NASH in rats. J. Med. Res. 2007, 36, 33-36.

102. Chen, X.; Cheng, X.W.; Xu, M.R. Protective effect of total flavones of hawthorn leaf on renal ischemia/reperfusion injury in rats. J. Appl. Clin. Pediatr. 2007, 22, 359-360.

103. Li, C.H.; Yang, Z.F.; Li, Z.X.; Ma, Y.; Zhang, L.P.; Zheng, C.B.; Qiu, W.W.; Wu, X.; Wang, X.; Li, H.; et al. Maslinic acid suppresses osteoclastogenesis and prevents ovariectomy-induced bone loss by regulating RANKL-mediated NF- $\mathrm{kB}$ and MAPK signaling pathways. J. Bone Mine. Res. 2011, 26, 644-656.

104. Tian, Q.F.; Guo, X.R.; Yin, W.J.; Yin, P.Z.; Dong, Y.L.; Hu, Z.M.; Li, J.X. Histological study of haw drink compound in protecting against experimental carbon disulfide toxic damage of relina. Chin. J. Ocular Fundus Dis. 1999, 15, 249-252.

105. Wei, J.Z.; Feng, L.; Bai, J.P.; Zhang, H.F.; Yang, L.Z.; Ma, C.G. The toxicology experiments of "semen cassiae hawthorn oat capsules". J. Shanxi Datong Univ. (Nat. Sci. Ed.) 2011, 25, 41-44.

(C) 2014 by the authors; licensee MDPI, Basel, Switzerland. This article is an open access article distributed under the terms and conditions of the Creative Commons Attribution license (http://creativecommons.org/licenses/by/3.0/). 